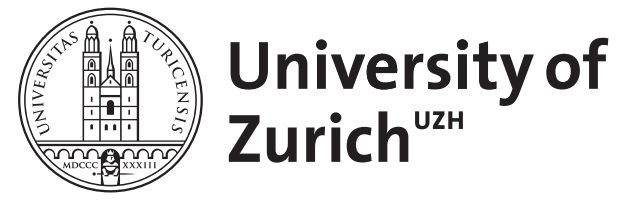

\title{
Aperiodic sequences and aperiodic geodesics
}

Schroeder, Viktor ; Weil, Steffen

\begin{abstract}
We introduce a quantitative condition on orbits of dynamical systems, which measures their aperiodicity. We show the existence of sequences in the Bernoulli shift and geodesics on closed hyperbolic manifolds which are as aperiodic as possible with respect to this condition.
\end{abstract}

DOI: https://doi.org/10.1017/etds.2013.2

Posted at the Zurich Open Repository and Archive, University of Zurich ZORA URL: https://doi.org/10.5167/uzh-92932

Journal Article

Published Version

Originally published at:

Schroeder, Viktor; Weil, Steffen (2014). Aperiodic sequences and aperiodic geodesics. Ergodic Theory and Dynamical Systems, 34(05):1699-1723.

DOI: https://doi.org/10.1017/etds.2013.2 


\section{Ergodic Theory and Dynamical Systems}

http://journals.cambridge.org/ETS

Additional services for Ergodic Theory and Dynamical Systems:

Email alerts: $\underline{\text { Click here }}$

Subscriptions: Click here

Commercial reprints: $\underline{\text { Click here }}$

Terms of use : $\underline{\text { Click here }}$

\section{Aperiodic sequences and aperiodic geodesics}

VIKTOR SCHROEDER and STEFFEN WEIL

Ergodic Theory and Dynamical Systems / FirstView Article / January 2014, pp 1 - 25

DOI: 10.1017/etds.2013.2, Published online: 14 March 2013

Link to this article: http://journals.cambridge.org/abstract_S0143385713000023

How to cite this article:

VIKTOR SCHROEDER and STEFFEN WEIL Aperiodic sequences and aperiodic geodesics.

Ergodic Theory and Dynamical Systems, Available on CJO 2013 doi:10.1017/etds.2013.2

Request Permissions : $\underline{\text { Click here }}$ 
Ergod. Th. \& Dynam. Sys. (First published online 2013), page 1 of $25^{*}$

doi:10.1017/etds.2013.2

(C) Cambridge University Press, 2013

*Provisional-final page numbers to be inserted when paper edition is published

\title{
Aperiodic sequences and aperiodic geodesics
}

\author{
VIKTOR SCHROEDER and STEFFEN WEIL \\ Institut für Mathematik, Mathematisch-naturwissenschaftliche Fakultät, \\ Universität Zürich, Winterthurerstrasse 190, 8057 Zürich, Switzerland \\ (e-mail:viktor.schroeder@math.uzh.ch,steffen.weil@math.uzh.ch)
}

(Received 4 June 2012 and accepted in revised form 27 December 2012)

Abstract. We introduce a quantitative condition on orbits of dynamical systems, which measures their aperiodicity. We show the existence of sequences in the Bernoulli shift and geodesics on closed hyperbolic manifolds which are as aperiodic as possible with respect to this condition.

\section{Main results}

In this section, we state our main results in the case of sequences in a finite alphabet and of geodesics in hyperbolic manifolds. Denote by $\mathbb{N}_{0}$ the natural numbers, including 0 , and let $\mathbb{N}=\mathbb{N}_{0} \backslash\{0\}$. Given a finite set $\mathcal{A}$ with $k \geq 2$ elements, let $\Sigma=\mathcal{A}^{\mathbb{Z}}$ be the set of biinfinite sequences in the alphabet $\mathcal{A}$, which we call words. By $[w(i) \ldots w(i+l)]$, denote the subword of $w \in \Sigma$ starting at time $i \in \mathbb{Z}$ and of length $l \in \mathbb{N}_{0}$. For a word $w \in \Sigma$, define the recurrence time $R_{w}^{i}: \mathbb{N}_{0} \rightarrow \mathbb{N} \cup\{\infty\}$ at time $i \in \mathbb{Z}$ by

$$
R_{w}^{i}(l)=\min \{s \geq 1:[w(i+s) \ldots w(i+s+l)]=[w(i) \ldots w(i+l)]\},
$$

i.e., the first instant when the subword $[w(i) \ldots w(i+l)]$ of $w$ is seen again, and by

$$
R_{w}(l):=\min \left\{R_{w}^{i}(l): i \in \mathbb{Z}\right\} .
$$

For a periodic word $w \in \Sigma$ with period $p \in \mathbb{N}$, i.e., $w(i)=w(i+p)$ for all $i \in \mathbb{Z}$, we have $R_{w}(l) \leq p$ for all $l \in \mathbb{N}_{0}$. Thus, if $R_{w}$ is unbounded, then $w$ is aperiodic and we view the growth rate of $R_{w}$ as a measure for the aperiodicity of the word $w$. Note that $R_{w}$ is nondecreasing and, by a trivial counting argument, we have $R_{w}(l) \leq k^{l+1}$ for every word $w$, in particular

$$
\limsup _{l \rightarrow \infty} \frac{1}{l} \ln R_{w}(l) \leq \ln (k) .
$$

One of our main results is the existence of words $w$ such that the growth rate is as near as possible to this bound. 
THEOREM 1.1. Let $\varphi: \mathbb{N}_{0} \rightarrow[0, \infty)$ be a non-decreasing function such that

$$
\limsup _{l \rightarrow \infty} \frac{1}{l} \ln (\varphi(l)) \leq \delta \ln (k)
$$

for some $0<\delta<1$. Then there exist $l_{0}=l_{0}(\varphi, k, \delta) \in \mathbb{N}_{0}$ and a word $w \in \Sigma$ such that, for every $l_{0} \leq l \in \mathbb{N}_{0}$, we have $R_{w}(l) \geq \varphi(l)$.

Now let $M$ be a closed $n$-dimensional hyperbolic manifold, where $n \geq 2$. Let $i_{M}>0$ denote the injectivity radius of $M$, and let $d$ be the Riemannian distance function on $M$. For a unit speed geodesic $\gamma: \mathbb{R} \rightarrow M$, we define the recurrence time $R_{\gamma}^{t_{0}}:[0, \infty) \rightarrow$ $\left[i_{M} / 2, \infty\right]$ at time $t_{0} \in \mathbb{R}$ by

$$
R_{\gamma}^{t_{0}}(l)=\inf \left\{s>i_{M} / 2: d\left(\gamma\left(t_{0}+t\right), \gamma\left(t_{0}+s+t\right)\right)<\frac{i_{M}}{2} \text { for all } 0 \leq t \leq l\right\},
$$

and

$$
R_{\gamma}(l):=\inf \left\{R_{\gamma}^{t_{0}}(l): t_{0} \in \mathbb{R}\right\} .
$$

If $\gamma$ is a periodic geodesic, then $R_{\gamma}$ is bounded and again one can view the growth rate of $R_{\gamma}$ as a measure for the aperiodicity of $\gamma$.

THEOREM 1.2. Let $\varphi:[0, \infty) \rightarrow[0, \infty)$ be a non-decreasing function such that

$$
\limsup _{l \rightarrow \infty} \frac{1}{l} \ln (\varphi(l)) \leq \delta(n-1)
$$

for some $0<\delta<1$. If $i_{M}>2 \ln (2)$, then there exist $l_{0}=l_{0}\left(\varphi, \delta, n, i_{M}\right) \geq 0$ and a unit speed geodesic $\gamma: \mathbb{R} \rightarrow M$ such that, for all $l \geq l_{0}$, we have $R_{\gamma}(l) \geq \varphi(l)$.

The theorems will later be shown in greater generality.

Remark. The bounds $\ln (k)$ and $n-1$ equal the topological entropies of the respective dynamical systems. Moreover, we believe that the assumption on the injectivity radius in Theorem 1.2 is not necessary. A version of this theorem is also true if $M$ is of strictly negative curvature. However, for the sake of clarity of the paper, we restrict these assumptions.

1.1. Organization of the paper. In $\S 2$, we will introduce the measure of aperiodcitiy for general dynamical systems and deduce immediate properties. In $\S \S 3$ and 4 , we examine two examples and state the main results, namely of the Bernoulli shift and the geodesic flow on a closed hyperbolic manifold. These will be proved in $\S 5$.

\section{2. $\quad F$-aperiodic points}

Let $(X, d)$ be a compact metric space and let $T: X \rightarrow X$ be a given continuous transformation. For $n \in \mathbb{N}_{0}$, let $T^{n}$ be the $n$-times composition of $T$ (where $T^{0}=i d_{X}$ ), and for a point $x \in X$, let $T^{n} x$ be the point in the orbit $\mathcal{T}(x):=\left\{T^{n} x\right\}_{n \in \mathbb{N}_{0}}$ of $x$ at time $n$. Moreover, let $\mu$ be a finite Borel measure on the Borel $\sigma$-algebra $\mathcal{B}$ of $(X, d)$ such that $T$ is measure-preserving; see [5]. 
A point $x \in X$ is called periodic (with respect to $T$ ) if there exists an integer $p \in \mathbb{N}$, called a period of $x$, such that $T^{p} x=x$. Denote by $\mathcal{P}_{T}$ the $T$-invariant set of $T$-periodic points of $X$. A point is called aperiodic if it is not periodic.

A point $x \in X$ is recurrent with respect to $T$ if for any $\varepsilon>0$, there exists $s=s(x, \varepsilon) \in \mathbb{N}$ such that $d\left(T^{s} x, x\right)<\varepsilon$. Periodic points are obviously recurrent. The set $\mathcal{R}_{T}$ of recurrent points is non-empty (see [6]) and $T$-invariant. However, $s\left(T^{i} x, \varepsilon\right)$ can differ from $s(x, \varepsilon)$ in general, unless $T$ is an isometry on its orbit $\mathcal{T}(x)$, i.e., $d\left(T^{i+s} x, T^{i} x\right)=d\left(T^{s} x, x\right)$ for all $i$ and $s \in \mathbb{N}_{0}$. We recall that by the Poincaré recurrence theorem, $\mu$-almost every point is recurrent.

In this paper, we give a quantitative version of recurrence and aperiodicity. Given a point $x \in X$ and a time $i \in \mathbb{N}_{0}$, we ask for a lower bound on the shift $s$ such that $T^{i+s} x$ is allowed to be $\varepsilon$-close to $T^{i} x$ :

Definition 2.1. For a non-increasing function $F:(0, \infty) \rightarrow[0, \infty)$, a point $x \in X$ is called $F$-aperiodic at time $i \in \mathbb{N}_{0}$ if, for every $\varepsilon>0$, whenever

$$
d\left(T^{i} x, T^{i+s} x\right)<\varepsilon
$$

for some $s \in \mathbb{N}$, then $s>F(\varepsilon)$. If $x$ is $F$-aperiodic at every time $i \in \mathbb{N}_{0}$, then it is called F-aperiodic.

We emphasize that although we called the condition ' $F$-aperiodic', a periodic point $x$ is $F$-aperiodic for a suitable bounded function $F$. However, if the function $F$ is unbounded, an $F$-aperiodic point must be aperiodic. Moreover, if $x$ is not recurrent, then it is easy to find an unbounded function $F$ such that $x$ is $F$-aperiodic, at least at time 0 .

Let $F:(0, \infty) \rightarrow[0, \infty)$ be a given non-increasing function. Clearly, if a nonincreasing function $\bar{F}$ satisfies $\bar{F}(s) \leq F(s)$ for all $s \in(0, \infty)$, then an $F$-aperiodic point is also $\bar{F}$-aperiodic. On the other hand, using the upper box $\operatorname{dimension}_{\operatorname{dim}_{B}}(X)$ for metric spaces, we obtain an upper bound on the growth rate (as $\varepsilon$ tends to 0 ) of functions $F$ such that an $F$-aperiodic point might exist. For $\varepsilon>0$ let $N(X, \varepsilon)$ denote the largest number of disjoint metric balls of radius $\varepsilon$. Then the upper box dimension [16] is given by

$$
\operatorname{dim}_{B}(X)=\limsup _{\varepsilon \rightarrow 0} \frac{\ln (N(X, \varepsilon))}{-\ln (\varepsilon)} .
$$

Lemma 2.2. Let $x$ be an $F$-aperiodic point. Then $\lim \sup _{\varepsilon \rightarrow 0}(\ln (F(\varepsilon)) / \ln (2 / \varepsilon)) \leq$ $\operatorname{dim}_{B}(X)$.

Proof. Let $\varepsilon>0$. If $B\left(T^{s_{1}} x, \varepsilon / 2\right) \cap B\left(T^{s_{2}} x, \varepsilon / 2\right) \neq \emptyset$ for some $0 \leq s_{1}<s_{2} \leq F(\varepsilon)$, we have $d\left(T^{s_{1}} x, T^{s_{2}} x\right)<\varepsilon_{0}$, which is impossible since $s_{2}-s_{1} \leq F\left(\varepsilon_{0}\right)$. Therefore, the metric balls $B\left(T^{s} x, \varepsilon / 2\right)$ must be disjoint for $s \leq F(\varepsilon)$. Hence, we have $F(\varepsilon) \leq$ $N(X, \varepsilon / 2)$.

Moreover, since $F$ is independent of the time $i \in \mathbb{N}_{0}$, the set $\mathcal{F}_{T} \subset X$ of $F$-aperiodic points is $T$-invariant. In the case when $(X, \mathcal{B}, \mu, T)$ is ergodic, $\mathcal{F}_{T}$ is either of full or of zero $\mu$-measure. When $\mathcal{P}_{T}$ is non-empty, this question is related to the distribution of periodic orbits. In fact, let $x_{0} \in \mathcal{P}_{T}$ be of minimal period $p_{0}$, and assume that $F(\varepsilon) \geq p_{0}$ 
for some $\varepsilon_{p_{0}}>0$. In the case when $F$ is continuous, we may choose $\varepsilon_{p_{0}}:=\sup \{\varepsilon>0$ : $\left.F(\varepsilon) \geq p_{0}\right\}$. Define the critical neighborhood of $x_{0}$ with respect to $F$ and $p_{0}$ by

$$
\mathcal{N}_{x_{0}}:=B\left(x_{0}, \varepsilon_{p_{0}} / 2\right) \cap T^{-p_{0}}\left(B\left(x_{0}, \varepsilon_{p_{0}} / 2\right)\right) .
$$

Whenever $x \in \mathcal{N}_{x_{0}}$, we have, by the triangle inequality, that $d\left(x, T^{p_{0}} x\right)<\varepsilon_{p_{0}}$, but $p_{0} \leq$ $F\left(\varepsilon_{p_{0}}\right)$. Thus, no point in $\mathcal{N}_{x_{0}}$ can be $F$-aperiodic, and we see that the orbit of an $F$ aperiodic point must avoid the critical neighborhoods of periodic points. If in addition $\mu\left(\mathcal{N}_{x_{0}}\right)>0$, then the set of $F$-aperiodic points cannot be of full, and must therefore be of zero, $\mu$-measure. Thus, we showed the following criterion.

Lemma 2.3. Assume $\mathcal{P}_{T} \neq \emptyset$, and let $x_{0}$ be a periodic point of period $p_{0}$ and $F(\varepsilon) \geq p_{0}$ for some $\varepsilon>0$. If $\mu$ is ergodic and positive on $\mathcal{N}_{x_{0}}$, then the set $\mathcal{F}_{T}$ has $\mu$-measure 0 .

In particular, this result is interesting for the systolic point $x_{0} \in \mathcal{P}_{T}$ of systolic period $p_{0} \in \mathbb{N}$, i.e., $x_{0}$ has minimal period $p_{0}$, and for every periodic point in $X$ of period $p$ we have $p \geq p_{0}$.

\section{LEMMA 2.4. F-aperiodicity is a closed condition.}

Proof. Let $\left\{x_{n}\right\}_{n \in \mathbb{N}}$ be a sequence of $F$-aperiodic points in $X$ converging to $x \in X$. Let $i$ and $s \in \mathbb{N}$ be fixed. For $\varepsilon>0$ such that $d\left(T^{i} x, T^{i+s} x\right)<\varepsilon$, let $d:=\frac{1}{2}(\varepsilon-$ $\left.d\left(T^{i} x, T^{i+s} x\right)\right)$. Since $T$ is continuous, there exists $N=N(i, s, d) \in \mathbb{N}_{0}$ such that, for all $n \geq N$, we have $d\left(T^{i} x, T^{i} x_{n}\right)<d$ and $d\left(T^{i+s} x, T^{i+s} x_{n}\right)<d$. From the triangle inequality, we obtain

$$
d\left(T^{i} x_{n}, T^{i+s} x_{n}\right) \leq d\left(T^{i} x_{n}, T^{i} x\right)+d\left(T^{i} x, T^{i+s} x\right)+d\left(T^{i+s} x, T^{i+s} x_{n}\right)<\varepsilon
$$

for $n \geq N$, so that $s>F(\varepsilon)$ since $x_{n}$ is $F$-aperiodic. Hence, $x$ is also $F$-aperiodic.

Finally, note that if $T$ acts as an isometry on the orbit $\mathcal{T}(x)$ of a point $x \in X$, then $x$ is $F$-aperiodic as soon as it is $F$-aperiodic at a given time. For instance, we consider the rotation on the circle as a motivating example.

Example 1 . Let $\mathbb{Z}$ act on $\mathbb{R}$ by translations, and let $X=\mathbb{R} / \mathbb{Z}$ be the compact quotient space with the induced metric $d$ obtained from the Euclidean metric. Given an irrational number $0<\alpha \in \mathbb{R} \backslash \mathbb{Q}$, we let $T=T_{\alpha}: X \rightarrow X$ be the automorphism induced by the translation $\tilde{T}: \mathbb{R} \rightarrow \mathbb{R}, \tilde{T}(x):=x+\alpha$. For $c>0$, we let $F_{c}:(0, \infty) \rightarrow[0, \infty), F_{c}(t)=c t^{-1}$. In fact, since $\operatorname{dim}_{B}(X)=1,-1$ is the optimal exponent as a result of Lemma 2.2. The point [0] is $F_{c}$-aperiodic if and only if every point $[x]$ is $F_{c}$-aperiodic, and hence $\mathcal{F}_{T}$ is either empty or $X$ itself. Moreover, since $T$ is an isometry, [0] is $F_{c}$-aperiodic as soon as it is $F_{c}$-aperiodic at time 0 . The question of for which $c$ and $\alpha$ there exist $F_{c}$-aperiodic points can be answered by classical Diophantine approximation; see, for instance, [1] for the following well-known results. Let $\mu$ be the Lebesgue measure on $\mathbb{R}$. For $\mu$-almost every $\alpha \in \mathbb{R} \backslash \mathbb{Q}$, we have $c_{0}(\alpha)=0$, where

$$
c_{0}(\alpha)=\inf \left\{c>0: \text { there exist infinitely many } p \in \mathbb{Z}, q \in \mathbb{N} \text { such that }\left|\alpha-\frac{p}{q}\right|<\frac{c}{q^{2}}\right\} .
$$


However, there exists a set of Hausdorff dimension one such that $c_{0}(\alpha)$ is positive. Such an $\alpha$ is called badly approximable. The supremum $\sup _{\alpha \in \mathbb{R} \backslash \mathbb{Q}} c_{0}(\alpha)$ of this set, called the Hurwitz constant, is equal to $1 / \sqrt{5}$ and attained at the golden ratio.

First, let $\alpha$ be such that $c_{0}(\alpha)=0$. Then, for $c>0$, we have, for infinitely many $p \in \mathbb{Z}$, $q \in \mathbb{N}$,

$$
\left|\tilde{T}^{q} 0-p\right|=|q \alpha-p|=q\left|\alpha-\frac{p}{q}\right|<c q^{-1} .
$$

Hence, $q \leq F_{c}\left(c q^{-1}\right)$, and we see that [0] is not $F_{c}$-aperiodic for any $c>0$. Thus, $\mathcal{F}_{T}$ is empty. In particular, this shows that for $c>1 / \sqrt{5}$, the set $\mathcal{F}_{T}$ is empty for every $T=T_{\alpha}$, $\alpha \in \mathbb{R} \backslash \mathbb{Q}$ irrational. However, for $\alpha$ a badly approximable number, we have $c_{0}(\alpha)>0$, and for $c<c_{0}(\alpha)$, there are only finitely many $p, q$ as in (2.2). Hence, we can choose some $0<\bar{c} \leq c_{0}(\alpha)$ such that [0] is $F_{\bar{c}}$-aperiodic, and therefore $\mathcal{F}_{T}=X$.

If we conversely assume that [0] is $F_{c}$-aperiodic, then, whenever $\left|\tilde{T}^{q} 0-p\right|<\varepsilon$ for some $\varepsilon>0$, we have $q>F_{c}(\varepsilon)=c / \varepsilon>c /(q|\alpha-p / q|)$. Thus, $|\alpha-p / q|>c / q^{2}$ for every $p \in \mathbb{Z}, q \in \mathbb{N}$, and $\alpha$ is necessarily a badly approximable number.

In the following, we are concerned with the examples of the Bernoulli shift and the geodesic flow on a closed hyperbolic manifold, where the question of the existence of $F$-aperiodic points is more delicate.

Remark. A somewhat orthogonal problem has been studied by many authors. For instance, [2] showed that the rate of recurrence can be quantified in the case when $X$ has finite Hausdorff dimension. More precisely, assume that the $\alpha$-dimensional Hausdorff measure $H_{\alpha}$ is $\sigma$-finite for some $\alpha>0$; then for $\mu$-almost every point $x \in X$, there exists a finite constant $c(x) \geq 0$ such that

$$
\liminf _{n \rightarrow \infty} n^{1 / \alpha} d\left(x, T^{n}(x)\right) \leq c(x) .
$$

Assume that there exists a point $x \in X$ which is $F$-aperiodic at time 0 for the function $F(\varepsilon)=c \cdot \varepsilon^{-\alpha}$, for some $c>0$ (compare with Lemma 2.2). Then it is not hard to show that for every $n>0$,

$$
n^{1 / \alpha} d\left(x, T^{n} x\right) \geq c^{1 / \alpha}
$$

The main point in our paper is that we study the recurrence for every point of the orbit and not only for the initial one.

\section{Sequences}

Let $\mathcal{A}$ be a finite set of $k \geq 2$ elements, which we call alphabet. Let $\Sigma^{+}=\{w: \mathbb{N} \rightarrow \mathcal{A}\}$ and $\Sigma=\{w: \mathbb{Z} \rightarrow \mathcal{A}\}$ be the set of two-sided sequences in symbols from $\mathcal{A}$. The elements of $\Sigma$ are called words. Given words $w$ and $\bar{w}$ in $\Sigma$, we let $a(w, \bar{w})=\max \{i \geq 0: w(i)=$ $\bar{w}(i)$ for $|j| \leq i\}$ for $w \neq \bar{w}$, and define $\bar{d}(w, \bar{w}):=2^{-a(w, \bar{w})}$, and $\bar{d}(w, w):=0$ otherwise. Let $T$ denote the shift operator acting on $\Sigma$, with $T(w)=\bar{w}$, where $\bar{w}(i)=w(i+1)$. Then $(\Sigma, \bar{d})$ is a compact metric space such that $T$ is a homeomorphism. Moreover, let $\mathcal{B}$ denote the product $\sigma$-algebra of the power set $\mathcal{P}(\mathcal{A})$ of $\mathcal{A}$ which equals the Borel $\sigma$-algebra 
of $(\Sigma, \bar{d})$. Let (the probability measure) $\mu=\prod_{\mathbb{Z}} \mu_{\mathcal{A}}$ be the infinite product measure of $\mathcal{B}$, where $\mu_{\mathcal{A}}$ is a probability measure on $(\mathcal{A}, \mathcal{P}(\mathcal{A}))$. Then the Bernoulli shift $(\Sigma, \mathcal{B}, \mu, T)$ is ergodic. For details, we refer to [5].

Note that by the definition of $\bar{d}$, two words are close if and only if the length of their subwords around position 0 on which they agree is large. In particular, if $w \in \mathcal{R}_{T}$, then, by recurrence applied to the word $T^{i} w$, for every length $l \in \mathbb{N}_{0}$, we can find an $s=s(i, l) \in \mathbb{N}$ such that $[w(i) \ldots w(i+l)]=[w(i+s) \ldots w(i+s+l)]$. In the case of sequences, it is suitable to reformulate $F$-aperiodicity as follows (see Proposition 3.2).

Definition 3.1. For a non-decreasing function $\varphi: \mathbb{N}_{0} \rightarrow[0, \infty)$, a word $w \in \Sigma$ is called $\varphi$-aperiodic at time $i \in \mathbb{Z}$, if, for every length $l \in \mathbb{N}_{0}$, whenever

$$
[w(i) \ldots w(i+l)]=[w(i+s) \ldots w(i+s+l)]
$$

for some shift $s \in \mathbb{N}$, then $s>\varphi(l)$. If $w$ is $\varphi$-aperiodic at every time $i \in \mathbb{Z}$, it is called $\varphi$-aperiodic.

A $\varphi$-aperiodic word $w \in \Sigma$ is $F$-aperiodic for the following function $F$.

PRoposition 3.2. A $\varphi$-aperiodic word $w \in \Sigma$ is $F$-aperiodic for $F(\varepsilon)=\varphi\left(-2\left\lceil\log _{2}(\varepsilon)\right\rceil\right)$. Conversely, an $F$-aperiodic word $w$ is $\varphi$-aperiodic for $\varphi(l)=F\left(2^{-(l-3) / 2}\right)$.

Proof. Let $i \in \mathbb{Z}$ and $s \in \mathbb{N}$. For every $l \in \mathbb{N}_{0}$ such that $\bar{d}\left(T^{i} w, T^{i+s} w\right) \leq 2^{-l}$, we have $[w(i-l) \ldots w(i+l)]=[w(i-l+s) \ldots w(i+s+l)]$. Thus, for $2^{-l}<\varepsilon \leq 2^{-(l-1)}$,

$$
s>\varphi(2 l)=\varphi\left(-2\left\lceil\log _{2}(\varepsilon)\right\rceil\right)=F(\varepsilon) .
$$

Since $F(\bar{\varepsilon}) \leq F(\varepsilon)$ for $\bar{\varepsilon} \geq \varepsilon$, the first implication follows.

Conversely, if $w$ is $F$-aperiodic, assume that $[w(i) \ldots w(i+l)]=[w(i+s) \ldots w(i+$ $s+l)]$ for $s \in \mathbb{N}, l \in \mathbb{N}_{0}$, and let $\bar{l}=l / 2$ if $l$ is even, and $\bar{l}=(l-1) / 2$ if $l$ is odd. Hence, $\bar{d}\left(T^{i+\bar{l}} w, T^{i+\bar{l}+s} w\right) \leq 2^{-\bar{l}}$, and for every $2^{-\bar{l}}<\varepsilon \leq 2^{-(\bar{l}-1)}$, we have

$$
s>F(\varepsilon) \geq F\left(2^{-(\bar{l}-1)}\right) \geq F\left(2^{-(l-3) / 2}\right)=\varphi(l) .
$$

This finishes the proof.

If a $\varphi$-aperiodic word contains a periodic subword of infinite length, then the function $\varphi$ is bounded, whereas if a word is $\varphi$-aperiodic for an unbounded function, the word must be aperiodic. We want to give some examples in order to make the definition more familiar, among them the prominent Morse-Thue sequence.

Example 2. First, let $a, b \in \mathcal{A}$. One checks that the (non-recurrent) words $w_{1}=$ $\ldots$. bbbaaa $\ldots$ and $w_{2}=\ldots$ abaabaaabaaaab $\ldots$ are $\varphi$-aperiodic only for a function $\varphi$ such that $1=s>\varphi(l)$, for all $l \in \mathbb{N}_{0}$. The orbits of $w_{1}$ and $w_{2}$ both come closer and closer to the periodic word . . a aa ... with respect to the metric $\bar{d}$. This is not the case for $\varphi$-aperiodic words when $\varphi$ is unbounded; see Proposition 3.4.

Consider the Morse-Thue recurrent sequence $w \in\{0,1\}^{\mathbb{Z}}$, which is determined as follows. Let $a_{0}=0, b_{0}=1$. Then, for $n \in \mathbb{N}_{0}$, let $a_{n+1}=a_{n} b_{n}$ and $b_{n+1}=b_{n} a_{n}$ be finite words of length $2^{n+1}-1$. Then $w$ is defined such that it satisfies $\left[w(0) \ldots w\left(2^{n}-\right.\right.$ $2)]=a_{n}$ and $[w(-n)]=[w(n-1)]$ for every $n \in \mathbb{N}$. In particular, $w$ contains the 
subwords $a_{n+2}=a_{n} b_{n} b_{n} a_{n}$. Hence, for every length $l=2^{n}-1, w$ contains subwords of the form $W W$, where $W$ has length $l$. A function $\varphi$ such that $w$ is $\varphi$-aperiodic must therefore be bounded by $\varphi\left(2^{n}-1\right) \leq 2^{n}-1$, for every $n \in \mathbb{N}$. On the other hand, there are no subwords of the form $W W a$, where $a$ is the first letter of a subword $W$ (see [11]). In other words, $w$ is overlap-free (which means that there are no subwords of the form $a W a W a$ for a finite subword $W$ and a letter $a$ ), from which it follows that there are even no subwords of the form $w W w W w$ for $w$ and $W$ finite subwords. Hence, we may choose $\varphi(l) \geq l$. We conclude that $w$ is at least $\varphi$-aperiodic for the function $\varphi(l)=l, l \in \mathbb{N}_{0}$.

The example shows that the set of $\varphi$-aperiodic words $\mathcal{F}_{T}=\mathcal{F}_{T}(\varphi)$ is non-empty for the unbounded function $\varphi(l)=l$ and, moreover, the Morse-Thue sequence gives an explicit example of such a word. However, let $a \in \mathcal{A}$ such that $\mu_{\mathcal{A}}(\{a\})>0$, and let $w=\ldots a a a \ldots$ be a periodic word which is of systolic period 1. Moreover, $\mu$ is positive on the critical neighborhood of $w$ and hence, by Lemma $2.3, \mathcal{F}_{T}$ is of zero $\mu$-measure unless $\varphi$ is strictly bounded by 1 .

Our main result for sequences is the following. It will be proved in $\S 5$.

THEOREM 3.3. Let $\varphi: \mathbb{N}_{0} \rightarrow[0, \infty)$ be a non-decreasing unbounded function such that there exists $c \in(1, k)$ satisfying

$$
k-\lfloor\varphi(0)\rfloor-\sum_{l=1}^{\infty} \frac{\lfloor\varphi(l)\rfloor-\lfloor\varphi(l-1)\rfloor}{c^{l}} \geq c,
$$

where $L \cdot\rfloor$ denotes the integer part. Then there exists a $\varphi$-aperiodic word in $\Sigma$.

Remark. The condition is satisfied for the following set of parameters.

(1) $\quad k \geq 4$, then $\varphi(l)=l$ satisfies (3.2) for $c=2$.

(2) $k \geq 5$, then $\varphi(l)=2^{l}$ satisfies (3.2) for $c=3$.

(3) $k \geq 2,0<\delta<1$ and $k^{\delta}<c<k$, then there exists $l_{0}=l_{0}(k, \delta, c) \in \mathbb{N}_{0}$ such that

$$
\varphi(l)= \begin{cases}0 & \text { for } l \leq l_{0}, \\ k^{\delta l} & \text { for } l>l_{0},\end{cases}
$$

satisfies (3.2).

Note that if a word $w$ is $\varphi$-aperiodic, then $R_{w}(l)>\varphi(l)$ for every $l \in \mathbb{N}_{0}$, where $R_{w}$ is the recurrence time introduced in $\S 1$. Theorem 1.1 is hence a corollary of Theorem 3.3.

Proof of Theorem 1.1. By condition (1.1), for every $\varepsilon_{0}>0$, there exists $l_{1}=l_{1}\left(\varepsilon_{0}\right) \in \mathbb{N}$ such that for all $l \geq l_{1}$,

$$
\frac{1}{l} \ln (\varphi(l)) \leq \delta \ln (k)\left(1+\varepsilon_{0}\right)
$$

Since $\delta<1$, we let $\varepsilon_{0}>0$ such that $\tilde{\delta}=\left(1+\varepsilon_{0}\right) \delta<1$. Then $\varphi(l) \leq k^{\tilde{\delta} l}$ for $l \geq l_{1}$. If we take $c:=\left(k-k^{\tilde{\delta}}\right) / 2$, then, by (3.3), there exists $l_{2}=l_{2}(k, \tilde{\delta})$ such that condition (3.2) is satisfied for the function $\bar{\varphi}(l):=k^{\tilde{\delta} l}$ for $l>l_{2}$ and $\bar{\varphi}(l)=0$ for $l \leq l_{2}, l \in \mathbb{N}_{0}$. Theorem 3.3 implies the existence of a $\bar{\varphi}$-aperiodic word $w \in \Sigma$. Thus, setting $l_{0}:=\max \left\{l_{1}, l_{2}\right\}+1$, we have that $\bar{\varphi}(l) \geq \varphi(l)$ for all $l \geq l_{0}$, and the claim follows. 
Remark. The critical function $\varphi$ for which $\varphi$-aperiodic words cannot exist is the function $\varphi(l)=k^{l+1}$. The critical exponent $\ln (k)$ equals the topological entropy of the system $(\Sigma, \bar{d}, T)$ (see [20]), and is optimal. To see that there is no $w \in \Sigma$ which is $\varphi$-aperiodic for a function $\varphi$ such that $\varphi(l) \geq k^{l+1}-1$ for some $l \in \mathbb{N}_{0}$, fix a subword $[w(1) \ldots w(1+l)]$ of any $w \in \Sigma$. Inductively, one shows that at each step $1 \leq s \leq \varphi(l)$, one has at most $k^{l+1}-s$ possibilities to choose a subword $[w(1+s) \ldots w(1+s+l)]$ such that $w$ stays $\varphi$-aperiodic. Then, at step $s=k^{l+1}$, there is no choice left such that $w$ is $\varphi$-aperiodic.

Remark. Let $\Sigma^{+}(m)=\{w:\{1, \ldots, m\} \rightarrow \mathcal{A}\}$ be the set of words of length $m$ in $\mathcal{A}$, and $\mathcal{W}^{g}(m) \subset \Sigma^{+}(m)$ be the set of good words of length $m$ which satisfy (3.1) for all $i, s \in \mathbb{N}$ and $l \in \mathbb{N}_{0}$ such that $i+s+l \leq m$. If $\varphi$ satisfies (3.2) with respect to the parameter $c>1$, we will see in the proof of Theorem 3.3 (see Lemma 5.6) that the good words $\mathcal{W}^{g}(m)$ increase in $m$ by the factor $c$. Thus, $\left|\mathcal{W}^{g}(m)\right| \geq c^{m}$, which is a lower bound on the asymptotic growth of $\left|\mathcal{W}^{g}(m)\right|$, where $|\cdot|$ denotes its cardinality.

We may reformulate the critical neighborhood of a periodic point given in (2.1) to the setting of $\varphi$-aperiodicity. Moreover, since $\mathcal{P}_{T}$ is dense in $\Sigma$, we can also give a sufficient condition on $\varphi$-aperiodicity in terms of periodic words. Therefore, for a non-decreasing unbounded function $\varphi: \mathbb{N}_{0} \rightarrow[0, \infty)$, we define a discrete form of a right inverse for $\varphi$ by $\ell: \mathbb{N} \rightarrow \mathbb{N}_{0}$,

$$
\ell(s)=\min \left\{j \in \mathbb{N}_{0}: \varphi(j) \geq s\right\},
$$

which is also non-decreasing and unbounded.

PROPOSITION 3.4. Let $\varphi: \mathbb{N}_{0} \rightarrow[0, \infty)$ be a non-decreasing unbounded function. If $w \in \Sigma$ is $\varphi$-aperiodic, then, for every periodic word $\bar{w} \in \Sigma$ of period $s$, and for all $i \in \mathbb{Z}$, we have

$$
\bar{d}\left(T^{i} w, \bar{w}\right)>2^{-(s+\ell(s)) / 2} .
$$

Conversely, if $\bar{d}\left(T^{i} w, \bar{w}\right)>2^{-(s+\ell(s)-1) / 2}$ for every periodic word $\bar{w}$ of period $s$ and all $i \in \mathbb{Z}$, then $w$ is $\varphi$-aperiodic.

Proof. If $w$ is $\varphi$-aperiodic, $w$ is aperiodic and there exists $m \in \mathbb{N}_{0}$ such that $\bar{d}\left(T^{i} w, \bar{w}\right)=$ $2^{-m}$, where we assume $2 m \geq s$ (otherwise the first statement follows). Hence, $[w(i-$ $m) \ldots w(i+m)]=[\bar{w}(-m) \ldots \bar{w}(m)]$, and we see that $[w(i-m) \ldots w(i-m+s+$ $(2 m-s))]=[w(i-m+s) \ldots w(i+m)]$. Thus, $s>\varphi(2 m-s)$ and $m<(s+\ell(s)) / 2$ from (5.1).

Conversely, assume that $[w(i) \ldots w(i+l)]=[w(i+s) \ldots w(i+s+l)]$ for $s \in \mathbb{N}$, $l \in \mathbb{N}_{0}$, and let $\bar{l}=(s+l) / 2$ if $s+l$ is even and $\bar{l}=(s+l-1) / 2$ if $s+l$ is odd. Moreover, let $\bar{w}$ be the periodic word of period $s$ such that $[\bar{w}(i) \ldots \bar{w}(i+s-1)]=$ $[w(i) \ldots w(i+s-1)]$. Thus, $2^{-\bar{l}} \geq d\left(T^{i+\bar{l}} w, T^{i+\bar{l}} \bar{w}\right)>2^{-(s+\ell(s)-1) / 2}$, and we see that $s+\ell(s)-1>2 \bar{l} \geq s+l-1$. Hence, $l<\ell(s)$ and, from (5.1), we have $s>\varphi(l)$.

Remark. Consider the overlap-free recurrence time $\tilde{R}_{w}^{0}: \mathbb{N}_{0} \rightarrow \mathbb{N}$ of the initial subword,

$$
\tilde{R}_{w}^{0}(l)=\min \{s>l:[w(s) \ldots w(s+l)]=[w(0) \ldots w(l)]\} .
$$


Clearly, $R_{w}(l) \leq R_{w}^{0}(l) \leq \tilde{R}_{w}^{0}(l)$ for $l \in \mathbb{N}_{0}$. Then it follows from [12] that, since the Bernoulli shift is ergodic, for $\mu$-almost all $w \in \Sigma$, the limit

$$
\lim _{l \rightarrow \infty} \frac{\ln \tilde{R}_{w}^{0}(l)}{l}
$$

exists and equals the measure-entropy $h_{\mu}(T)$.

\section{Geodesic flow on hyperbolic manifolds}

Let $M$ be a closed $n$-dimensional hyperbolic manifold, that is, a compact connected Riemannian manifold without boundary of constant negative curvature -1 , where $n \geq 2$. We denote by $d$ the distance function on $M$ and by $i_{M}>0$ the injectivity radius.

Let $S M$ be the unit tangent bundle of $M$ and $d^{S}$ the Sasaki-distance function on $S M$. For $v \in S M$, let $\gamma_{v}: \mathbb{R} \rightarrow M$ be the unit speed geodesic such that $\gamma_{v}^{\prime}(0)=v$. The geodesic flow $\phi^{t}: S M \rightarrow S M, t \in \mathbb{R}$, acts on the compact metric space $\left(S M, d^{S}\right)$ by diffeomorphisms, where $\phi^{t} v=\gamma_{v}^{\prime}(t)$. For details and background, we refer to [4].

A vector $v \in S M$ is periodic if there exists a $t>0$ such that $\phi^{t} v=v$, and $v$ is recurrent if for every $\varepsilon>0$, there exists $s>0$ such that $d^{S}\left(\phi^{s} v, v\right)<\varepsilon$. Denote by $\mathcal{P}_{\phi}$ and $\mathcal{R}_{\phi}$ the flow-invariant sets of periodic, respectively recurrent, vectors. Thus, if $v \in \mathcal{R}_{\phi}$, then for given $t \in \mathbb{R}, \varepsilon>0$, there exists $s=s(t, \varepsilon)$ such that $d^{S}\left(\phi^{t+s} v, \phi^{t} v\right)<\varepsilon$.

We now adjust the definitions of $F$-aperiodic and $\varphi$-aperiodic points to the setting of the geodesic flow.

Definition 4.1. Let $F:(0, \infty) \rightarrow[0, \infty)$ be a non-increasing function and $s_{0}>0$ be a constant, called the minimal shift. A vector $v \in S M$ is called $F$-aperiodic (with minimal shift $\left.s_{0}\right)$ at $t_{0} \in \mathbb{R}$ if, for every $\varepsilon>0$, whenever

$$
d^{S}\left(\phi^{t_{0}} v, \phi^{t_{0}+s} v\right)<\varepsilon
$$

for some shift $s>s_{0}$, then $s>F(\varepsilon)$. If $v$ is $F$-aperiodic at every time $t_{0}$, then $v$ is called $F$-aperiodic (with minimal shift $s_{0}$ ).

Note that in contrast to the discrete setting in $\S 2$ (where $s \in \mathbb{N}$, i.e., $s \geq 1$ ) we now have to specify the additional parameter $s_{0}$, since $d^{S}\left(\phi^{t_{0}} v, \phi^{t_{0}+s} v\right)=s$ for $s$ small enough.

We also have to generalize the notion of $\varphi$-aperiodicity. All geodesics will be assumed to be unit speed. Note that as in the case of the Bernoulli shift, two vectors in the Sasaki distance are very close if and only if the trajectories of the corresponding geodesics are close (in the Riemannian distance) to each other for a long time. Thus, we may reformulate $\varphi$-aperiodicity in terms of the fellow traveler length.

We introduce a second parameter, the distance constant $\varepsilon_{0}>0$.

Definition 4.2. Let $\varphi:[0, \infty) \rightarrow[0, \infty)$ be a non-decreasing function; let $0<\varepsilon_{0}<i_{M}$ and $s_{0} \geq \varepsilon_{0}$. A geodesic $\gamma: \mathbb{R} \rightarrow M$ is called $\varphi$-aperiodic at time $t_{0} \in \mathbb{R}$ if for every length $l>\varepsilon_{0}$, whenever

$$
d\left(\gamma\left(t_{0}+t\right), \gamma\left(t_{0}+s+t\right)\right)<\varepsilon_{0} \quad \text { for all } 0 \leq t \leq l
$$

for some shift $s>s_{0}$, then $s>\varphi(l)$. If $\gamma$ is $\varphi$-aperiodic at every time $t_{0}$, it is called $\varphi$ aperiodic (with parameters $\left(s_{0}, \varepsilon_{0}\right)$ ). 
The geodesic flow on compact hyperbolic manifolds is ergodic with respect to the Liouville measure $\mu$ (on the Borel $\sigma$-algebra of $S M$ ). A systole of $M$ has length $2 i_{M}$, which equals the systolic period. For a non-decreasing function $\varphi$, let $\mathcal{F}_{\phi}$ be the set of $\varphi$-aperiodic geodesics (with respect to $\left(s_{0}, \varepsilon_{0}\right)$ ), which is invariant under the geodesic flow $\phi^{t}$. Since $\mu$ is positive on open sets, one can show, as in Lemma 2.3, that the set $\mathcal{F}_{\phi}$ is of zero $\mu$-measure if and only if $\varphi$ is not bounded by either $s_{0}$ or $2 i_{M}-\varepsilon_{0}$.

The main result of this section is the following, which will be proved in $\S 5$.

THEOREM 4.3. Assume that $i_{M}>\ln (2)$, and let $\varepsilon_{0}>0$ such that $\ln (2)+\varepsilon_{0}<i_{M}$. Let

$$
\varphi_{\delta}(l)=e^{\delta(n-1) l},
$$

where $0<\delta<1$. Then there exists a minimal length $l_{0}=l_{0}\left(\delta, i_{M}, n, \varepsilon_{0}\right)$ and a geodesic $\gamma: \mathbb{R} \rightarrow M$, which satisfies, for every $t_{0} \in \mathbb{R}$ and all $l \geq l_{0}$, whenever

$$
d\left(\gamma\left(t_{0}+t\right), \gamma\left(t_{0}+s+t\right)\right)<\varepsilon_{0} \quad \text { for all } 0 \leq t \leq l
$$

for some shift $s>\varepsilon_{0}$, then $s>\varphi_{\delta}(l)$.

Note that for $\varepsilon_{0}=i_{M} / 2$, if a geodesic $\gamma: \mathbb{R} \rightarrow M$ satisfies (4.1), then $R_{\gamma}(l) \geq \varphi_{\delta}(l)$, for all $l \geq l_{0}$, where $R_{\gamma}$ is the recurrence time introduced in $\S 1$. Theorem 1.2 is therefore a corollary of Theorem 4.3.

Proof of Theorem 1.2. By (1.2), there exists, for every $\tau>0$, some $l_{1}=l_{1}(\tau) \geq 0$ such that for all $l \geq l_{1}$, we have

$$
\varphi(l) \leq e^{(1+\tau)(n-1) \delta l} .
$$

Since $\delta<1$, we let $\tau_{0}>0$ such that $\bar{\delta}:=\left(1+\tau_{0}\right) \delta<1$. From Theorem 4.3, for $\varepsilon_{0}=i_{M} / 2$, there exists $l_{2}=l_{2}\left(\bar{\delta}, i_{M}, n\right)$ and a geodesic $\gamma: \mathbb{R} \rightarrow M$ such that for every $t_{0} \in \mathbb{R}$ and $l \geq l_{2}$, whenever

$$
d\left(\gamma\left(t_{0}+t\right), \gamma\left(t_{0}+s+t\right)\right)<\frac{i_{M}}{2} \quad \text { for all } 0 \leq t \leq l,
$$

for some shift $s>i_{M} / 2$, then $s>e^{\bar{\delta}(n-1) l}$. If we set $l_{0}:=\max \left\{l_{1}, l_{2}\right\}$, then $s>e^{\bar{\delta}(n-1) l} \geq$ $\varphi(l)$, whenever $l \geq l_{0}$, and the proof is finished.

In order to prove Theorem 4.3, we discretize our geodesics. Therefore, we need a third parameter, the discretization constant, $r_{0}>0$. For a geodesic $\gamma: \mathbb{R} \rightarrow M$, we consider the discrete geodesic

$$
\bar{\gamma}: \mathbb{Z} \rightarrow M, \quad \bar{\gamma}(i):=\gamma\left(i \cdot r_{0}\right)
$$

Definition 4.4. (Discrete definition) Let $\bar{\varphi}: \mathbb{N}_{0} \rightarrow[0, \infty)$ be a non-decreasing function, and let the parameters $\left(\bar{s}_{0}, \bar{\varepsilon}_{0}, r_{0}\right)$ be given, where $\bar{s}_{0} \in \mathbb{N}_{0}, 0<\bar{\varepsilon}_{0}<i_{M}$ and $0<r_{0}<\bar{\varepsilon}_{0}$. A discrete geodesic $\bar{\gamma}: \mathbb{Z} \rightarrow M$ is called $\bar{\varphi}$-aperiodic at time $i \in \mathbb{Z}$ if, for $l \in \mathbb{N}$, whenever

$$
d(\bar{\gamma}(i+j), \bar{\gamma}(i+s+j))<\bar{\varepsilon}_{0} \quad \text { for all } j \in\{0, \ldots, l\}
$$

for some shift $s>\bar{s}_{0}$, then $s>\bar{\varphi}(l) . \bar{\gamma}$ is called $\bar{\varphi}$-aperiodic (with parameters $\left(\bar{s}_{0}, \bar{\varepsilon}_{0}, r_{0}\right)$ ) if it is $\bar{\varphi}$-aperiodic at every time $i \in \mathbb{Z}$. 
Note that, given a $\bar{\varphi}$-aperiodic geodesic $\bar{\gamma}: \mathbb{Z} \rightarrow M$ (with the parameters $\left(\bar{s}_{0}, \bar{\varepsilon}_{0}, r_{0}\right)$ ), the corresponding geodesic $\gamma: \mathbb{R} \rightarrow M$ is continuously $\varphi$-aperiodic in the following way.

LEMMA 4.5. For a non-decreasing function $\bar{\varphi}:[0, \infty) \rightarrow[0, \infty)$ and the parameters $\left(\bar{s}_{0}, \bar{\varepsilon}_{0}, r_{0}\right)$, let $\bar{\gamma}: \mathbb{Z} \rightarrow M$ be a $\left.\bar{\varphi}\right|_{\mathbb{N}_{0}}$-aperiodic geodesic. For $r_{0} \leq l \in \mathbb{R}$, define

$$
\varphi(l):=r_{0} \cdot \bar{\varphi}\left(\frac{l-r_{0}}{r_{0}}\right)-r_{0} .
$$

Then $\gamma$ is $\varphi$-aperiodic with respect to the minimal shift $s_{0}=\left(\bar{s}_{0}+1\right) r_{0}$ and the distance constant $\varepsilon_{0}=\bar{\varepsilon}_{0}-r_{0}>0$.

Conversely, if $\gamma: \mathbb{R} \rightarrow M$ is $\varphi$-aperiodic with parameters $\left(s_{0}, \varepsilon_{0}\right)$, then, for $r_{0}<\varepsilon_{0}$, let

$$
\bar{\varphi}(l):=\varphi\left(l \cdot r_{0}\right) / r_{0} .
$$

Then $\bar{\gamma}: \mathbb{Z} \rightarrow M$ is $\bar{\varphi}$-aperiodic with parameters $\left(\left\lceil s_{0} / r_{0}\right\rceil, \varepsilon_{0}, r_{0}\right)$.

Proof. For $t_{0} \in \mathbb{R}, L \geq r_{0}$ and $s>\left(\bar{s}_{0}+1\right) r_{0}$, assume that $d\left(\gamma\left(t_{0}+t\right), \gamma\left(t_{0}+s+t\right)\right)<$ $\varepsilon_{0}$ for all $0 \leq t \leq L$. If we set $i:=\left\lceil t_{0} / r_{0}\right\rceil$ and $i+\bar{s}:=\left\lceil\left(t_{0}+s\right) / r_{0}\right\rceil$, whereas $l:=\left\lfloor L / r_{0}\right\rfloor$, we have $i, l \geq 1$ and $\bar{s}>\bar{s}_{0}$. Then, since $\varepsilon_{0}=\bar{\varepsilon}_{0}-r_{0}<i_{M}$ and the distance function is locally convex, one checks by the triangle inequality that $d(\bar{\gamma}(i), \bar{\gamma}(i+\bar{s}))<\bar{\varepsilon}_{0}$ and $d(\bar{\gamma}(i+l), \bar{\gamma}(i+\bar{s}+l))<\bar{\varepsilon}_{0}$. In particular, $d(\bar{\gamma}(i+j), \bar{\gamma}(i+\bar{s}+j))<\bar{\varepsilon}_{0}$ for all $0 \leq$ $j \leq l$. Thus, $\bar{s}>\bar{\varphi}(l)$, so that

$$
s \geq(\bar{s}-1) r_{0}>(\bar{\varphi}(l)-1) r_{0} \geq\left(\bar{\varphi}\left(\frac{L}{r_{0}}-1\right)-1\right) r_{0}=\varphi(L),
$$

since $(l+1) r_{0} \geq L$. This finishes the first part of the lemma. The second part follows analogously.

In terms of Lemma 4.5, we are left with stating the existence theorem for discrete $\bar{\varphi}$ aperiodic geodesics. Recall that for an unbounded function $\bar{\varphi}$, we defined its discrete right inverse $\bar{\ell}: \mathbb{N} \rightarrow \mathbb{N}_{0}$ in (3.4), which is also non-decreasing and unbounded.

THEOREM 4.6. Let $\bar{\varphi}: \mathbb{N}_{0} \rightarrow[0, \infty)$ be a non-decreasing, unbounded function. Assume that $\ln (2)<r_{0}<\bar{\varepsilon}_{0}<i_{M}$ and $\bar{s}_{0} \in \mathbb{N}_{0}$ such that, for all $l \geq \bar{s}_{0}$,

$$
\lfloor\bar{\varphi}(l)\rfloor>l \quad \text { and } \quad \bar{\ell}\left(\bar{s}_{0}\right) \geq 1,
$$

and, moreover, that there exists a constant $c \in\left(1,2^{n-1}\right)$ such that

$$
2^{n-1}-\bar{c} \cdot \sum_{l=\bar{\ell}\left(\bar{s}_{0}\right)}^{\infty} \frac{\lfloor\bar{\varphi}(l)\rfloor-\lfloor\bar{\varphi}(l-1)\rfloor}{c^{l}} \geq c,
$$

where $\bar{c}$ is an explicit constant depending only on $n$ and $i_{M}$. Then there exist a $\bar{\varphi}$-aperiodic geodesic $\gamma: \mathbb{Z} \rightarrow M$ with the parameters $\left(\bar{s}_{0}, \bar{\varepsilon}_{0}, r_{0}\right)$.

Remark. Since $\bar{\ell}$ is unbounded, condition (4.4) depends again essentially on the convergence of the sum in (4.4). For instance, let $\delta \in(0,1)$ and define $\bar{\varphi}(l)=2^{\delta(n-1) l}$, and let $c \in\left(2^{\delta(n-1)}, 2^{n-1}\right)$. Then, since $\bar{\ell}(s)=\lceil(1 / \delta(n-1) \ln (2)) \ln (s)\rceil$ for $s \geq 0$, there exists a minimal shift $\bar{s}_{0}=\bar{s}_{0}(n, \delta, \bar{c}, c)$ such that (4.3) and (4.4) are satisfied. 
The constant $\bar{c}$ of condition (4.4) can in fact be sharpened to be also dependent on $\bar{s}_{0}$, in which case it is strictly decreasing in $\bar{s}_{0}$. It will be explicitly defined in the proof of Claim 5.12. We may give a rough upper bound on $\bar{c}$ which is independent of $\bar{s}_{0}$ by

$$
\bar{c} \leq\left\lceil\left(3 \cosh \left(i_{M}\right) \sqrt{n+1}\right)^{n-1}\right\rceil\left\lceil\frac{\int_{0}^{5 i_{M}+4 \ln (\sqrt{n+1} / 2)} \sinh (t)^{n-1} d t}{\int_{0}^{i_{M} / 2} \sinh (t)^{n-1} d t}\right\rceil .
$$

The lower bound $\ln (2)$ on the injectivity radius is necessary for the proof. However, we believe that the result should be valid without this bound. Moreover, a version of Theorem 4.6 remains true for $M$ a closed $n$-dimensional Riemannian manifold of negative sectional curvature.

Remark. Again, the critical function $\varphi$ such that $\varphi$-aperiodic geodesics might or might not exist seems to be the function $\varphi(s)=e^{(n-1) s}$ and the critical exponent $n-1$ equals the topological entropy of $\left(S M, \phi^{t}\right)$.

Lemma 2.2 gives an upper bound on the growth rate of non-increasing functions $F:(0, \infty) \rightarrow(0, \infty)$ for which $F$-aperiodic geodesics can exist. In fact, since $S M$ is a $(2 n-1)$-dimensional manifold, its box dimension is $2 n-1$. Discretizing $\phi^{t}$ by the time $t_{0}$-map $\phi^{t_{0}}$, where $t_{0}=t_{0}\left(i_{M}\right)>0$ is sufficiently small, gives the upper bound

$$
\limsup _{\varepsilon \rightarrow 0} \frac{\ln (F(\varepsilon))}{\ln (2 / \varepsilon)} \leq 2 n-1 .
$$

Remark. For a closed geodesic $\alpha: \mathbb{R} \rightarrow M$, let $\mathcal{N}_{\varepsilon_{0}}(\alpha)$ be the (closed) $\varepsilon_{0} / 2$-neighborhood of $\alpha$ in $M$, where $\varepsilon_{0}>0$ is sufficiently small. When a geodesic $\gamma: \mathbb{R} \rightarrow M$ enters $\mathcal{N}_{\varepsilon_{0}}(\alpha)$ at time $t_{0}$, let $\mathfrak{p}_{\alpha}\left(\gamma, t_{0}\right)$ be the penetration length of $\gamma$ in $\alpha$ at time $t_{0}$, that is, the maximal length $L \in[0, \infty]$ of an interval $I, t_{0} \in I$, such that $\gamma(t) \in \mathcal{N}_{\varepsilon_{0}}(\alpha)$ for all $t \in I$. Set $\mathfrak{p}_{\alpha}\left(\gamma, t_{0}\right)=0$ if $\gamma\left(t_{0}\right) \notin \mathcal{N}_{\varepsilon_{0}}(\alpha)$. Then, by [10], for $\mu$-almost every $v \in S M$, the limit

$$
\limsup _{t \rightarrow \infty} \frac{\mathfrak{p}_{\alpha}(\gamma, t)}{\ln (t)}
$$

exists and equals $1 /(n-1)$.

Moreover, the penetration length reflects the depth in which $\gamma$ enters the neighborhood $\mathcal{N}_{\varepsilon_{0}}(\alpha)$. The study of depths or penetration lengths in an adequate convex set of negatively curved manifolds, such as the $\varepsilon$-neighborhood of totally geodesic embedded submanifold or the cusp neighborhood of a finite-volume hyperbolic manifold, leads to the theory of diophantine approximation in negatively curved manifolds; see, for instance, $[7,9,10,13-$ $15,17,18$, to give only a short and incomplete list. In general, a sequence of depths or penetration lengths and times of $\gamma$ in these convex sets reflects 'how well $\gamma$ is approximated', where $\gamma$ is called badly approximable if any such sequence is bounded; see $[\mathbf{9}, \mathbf{1 0}]$.

Now let $\gamma$ be a $\varphi$-aperiodic geodesic ( $\varphi$ unbounded) with respect to the parameters $s_{0}$ and $\varepsilon_{0}$, and let $\alpha$ be any closed geodesic in $M$. Then, it can be seen that the penetration lengths of $\gamma$ in $\mathcal{N}_{\varepsilon_{0}}(\alpha)$ are bounded by a constant depending only on $\varphi, \varepsilon_{0}$ and the length of $\alpha$ (and $s_{0}$ respectively). Therefore, the notion of $\varphi$-aperiodicity is linked to bad approximation; recall also Example 1. In particular, the limit of (4.6) equals 0 for $\gamma$. 


\section{Proofs}

Let $\varphi: \mathbb{N}_{0} \rightarrow[0, \infty)$ be a non-decreasing unbounded function. Recall the definition of the function $\ell: \mathbb{N} \rightarrow \mathbb{N}_{0}$ given by

$$
\ell(s)=\min \left\{j \in \mathbb{N}_{0}: \varphi(j) \geq s\right\}
$$

see (3.4). The following properties hold: $\ell$ is non-decreasing and for $s$ and $l \in \mathbb{N}_{0}$, we have

$$
\begin{gathered}
\varphi(\ell(s)) \geq s, \\
l<\ell(s) \Longleftrightarrow \varphi(l)<s, \\
l \geq \ell(s) \Longleftrightarrow \varphi(l) \geq s .
\end{gathered}
$$

Proof. For the first property, clearly $\varphi(\min \{j: \varphi(j) \geq s\}) \geq s$. Let $l<\ell(s)$, and assume $s \leq \varphi(l)$. Then $\ell(s)=\min \{j: \varphi(j) \geq s\} \leq l$; a contradiction. If $s>\varphi(l)$, then $\ell(s)=$ $\min \{j: \varphi(j) \geq s\}>l$, and if $\varphi(l) \geq s$, then $\ell(j)=\min \{j: \varphi(j) \geq s\} \leq l$. Also, if $l \geq \ell(s)$, then $\varphi(l) \geq \varphi(\ell(s)) \geq s$.

5.1. Proof of Theorem 3.3. Recall that $\Sigma^{+}(m)=\{w:\{1, \ldots, m\} \rightarrow \mathcal{A}\}$ is the set of words of length $m-1$. We consider $\Sigma^{+}(m)$ to be a subset of $\Sigma^{+}=\mathcal{A}^{\mathbb{N}}$ (for example, by extending an element $w \in \Sigma^{+}(m)$ to an element $\bar{w} \in \Sigma^{+}$by setting $\bar{w}(i)=a$ for all $i>m$, where $a \in \mathcal{A}$ is fixed).

Definition 5.1. Let $m \in \mathbb{N}$. A word $w \in \Sigma^{+}(m)$ is called $\varphi$-aperiodic if, for all $i, s \in \mathbb{N}$ and $l \in \mathbb{N}_{0}$ such that $i+s+l \leq m$, whenever

$$
[w(i) \ldots w(i+l)]=[w(i+s) \ldots w(i+s+l)],
$$

we have $s>\varphi(l)$.

Let $l_{0}:=\min \left\{j \in \mathbb{N}_{0} \cup\{-1\}: \varphi(j+1) \neq 0\right\}$, and note that $\ell(s)>l_{0}$ for all $s \in \mathbb{N}$. For $m \in \mathbb{N}$, define the admissible set by

$$
A(m):=\{(i, s) \in \mathbb{N} \times \mathbb{N}: i+s+\ell(s)=m\},
$$

if $m \geq m_{0}:=2+\ell(1)>2+l_{0}$, and let $A(m)$ be empty for $m<m_{0}$. Then, for $(i, s) \in$ $A(m)$, where $m \geq m_{0}$, we define the sets

$$
C_{i s}:=\left\{w \in \Sigma^{+}(m):[w(i) \ldots w(i+\ell(s))] \neq[w(i+s) \ldots w(i+s+\ell(s))]\right\},
$$

called conditions.

Remark. Note that $s>\varphi(\ell(s)-1)$ for $\ell(s)>0$, but $s \leq \varphi(\ell(s))$. Therefore, $\ell(s)$ determines the critical length of a given shift $s$ with respect to $\varphi$.

For $w \in \Sigma^{+}(m)$ and $1 \leq n \leq m$, let $\left.w\right|_{n}:=[w(1) \ldots w(n)] \in \Sigma^{+}(n)$. This leads to the reformulation of $\varphi$-aperiodic words.

Lemma 5.2. For $m<m_{0}$, every word $w \in \Sigma^{+}(m)$ is $\varphi$-aperiodic. For $m \geq m_{0}$, a word $w \in \Sigma^{+}(m)$ is $\varphi$-aperiodic if and only if, for all $n \leq m$ and all $(i, s) \in A(n)$, we have $\left.w\right|_{n} \in C_{i s}$. 
Proof. First, let $m<m_{0}$. Then, for every $i, s \in \mathbb{N}, l \in \mathbb{N}_{0}$ such that $i+s+l \leq m<$ $2+\ell(1)$, we have, in particular, $l<\ell(1)$. Equivalently, $\varphi(l)<1$, so that $s>\varphi(l)$ and every word $[w(1) \ldots w(m)]$ follows to be $\varphi$-aperiodic.

Now let $m \geq m_{0}$. Let $w$ be $\varphi$-aperiodic and assume $\left.w\right|_{n} \notin C_{i s}$ for some $i$ and $s$ in $\mathbb{N}$ such that $i+s+\ell(j)=n \leq m$. Then

$$
[w(i) \ldots w(i+\ell(s))]=[w(i+s) \ldots w(i+s+\ell(s))]
$$

and by (3.1), we have $s>\varphi(\ell(s))$; a contradiction to $\varphi(\ell(s)) \geq s$.

Conversely, assume that $w$ is not $\varphi$-aperiodic. Then there are $i, s \in \mathbb{N}$ and $l \in \mathbb{N}_{0}$ such that $i+s+l \leq m$ and

$$
[w(i) \ldots w(i+l)]=[w(i+s) \ldots w(i+s+l)],
$$

with $s \leq \varphi(l)$. This implies that $\ell(s) \leq l$ and, in particular,

$$
[w(i) \ldots w(i+\ell(s))]=[w(i+s) \ldots w(i+s+\ell(s))] .
$$

Hence, it follows that $\left.w\right|_{n} \notin C_{i s}$, since $i+s+\ell(s)=n \leq m$, so that $(i, s) \in A(n)$.

Note that by the same arguments as in the previous proof, a word $w \in \Sigma^{+}$is $\varphi$-aperiodic if and only if, for all $n \geq m_{0}$ and all $(i, s) \in A(n)$, we have $\left.w\right|_{n} \in C_{i s}$.

For $m \in \mathbb{N}$ such that $m \geq m_{0}$, the set of good words of length $m$ is therefore given by

$$
\mathcal{W}^{g}(m)=\left\{w \in \Sigma^{+}(m):\left.w\right|_{n} \in C_{i s} \text { for all }(i, s) \in A(n) \text { where } n \leq m\right\},
$$

and by $\mathcal{W}^{g}(m)=\Sigma^{+}(m)$ otherwise. Let

$$
\mathcal{C}_{m}=\left\{C_{i s}:(i, s) \in A(m)\right\}
$$

be the set of conditions at place $m$ which is empty if and only if $m<m_{0}$. Clearly, if $w \in \mathcal{W}^{g}(m)$, then $\left.w\right|_{n} \in \mathcal{W}^{g}(n)$ for $n \leq m$.

LEMMA 5.3. For $m \in \mathbb{N}$,

$$
\left|\mathcal{W}^{g}(m+1)\right| \geq k \cdot\left|\mathcal{W}^{g}(m)\right|-\sum_{C_{i s} \in \mathcal{C}_{m+1}}\left|\mathcal{W}^{g}(i+s-1)\right| .
$$

Proof. If $m+1<m_{0}$, then $\mathcal{C}_{m+1}$ is empty and the claim follows. Hence, let $m+1 \geq m_{0}$. Set $L=\left\{w \in \Sigma^{+}(m+1):\left.w\right|_{m} \in \mathcal{W}^{g}(m)\right\}$. Then

$$
\mathcal{W}^{g}(m+1)=L \cap\left(\bigcap_{C_{i s} \in \mathcal{C}_{m+1}} C_{i s}\right)=L \backslash\left(\bigcup_{C_{i s} \in \mathcal{C}_{m+1}}\left(L \cap C_{i s}^{C}\right)\right),
$$

where $C_{i s}^{C}$ denotes the complement of $C_{i s}$. Fix some condition $C_{i s} \in \mathcal{C}_{m+1}$. Since $|L|=k \cdot\left|\mathcal{W}^{g}(m)\right|$, the lemma follows from the following claim.

Claim 5.4. $\left|L \cap C_{i s}^{C}\right| \leq\left|\mathcal{W}^{g}(i+s-1)\right|$.

Proof. If $Q:=\left\{\left.w\right|_{i+s-1} \in \Sigma^{+}(i+s-1): w \in L\right\}$, then, clearly, $|Q| \leq\left|\mathcal{W}^{g}(i+s-1)\right|$. Decompose $L$ into $L=\bigcup_{q \in Q} L_{q}$, where $L_{q}=\left\{w \in L:\left.w\right|_{i+s-1}=q\right\}$. By definition, different elements in $L_{q}$ have different subwords $[w(i+s) \ldots w(m+1)]$ and, moreover,

$$
L \cap C_{i s}^{C}=\{w \in L:[w(i) \ldots w(i+\ell(s))]=[w(i+s) \ldots w(m+1)]\} .
$$


Hence, if $s>\ell(s)$, then an element $w$ of $L_{q}$, which is also in $C_{i s}^{C}$, is uniquely determined by $q$, which means $w$ is of the form $\left.w\right|_{i+s-1}=q$, and

$$
[w(i+s) \ldots w(m+1)]=[q(i) \ldots q(i+\ell(s))] .
$$

If $s \leq \ell(s)$, then one inductively checks that a word $w$ in $L_{q} \cap C_{i s}^{C}$ is of the form $\left.w\right|_{i+s-1}=q$,

$$
\begin{aligned}
{[w(i+j s) \ldots w(i+(j+1) s-1)] } & =[w(i+(j-1) j) \ldots w(i+j s-1)]=\cdots= \\
& =[w(i) \ldots w(i+s-1)]=[q(i) \ldots q(i+s-1)],
\end{aligned}
$$

for $1 \leq j \leq j_{0}$, where $j_{0}$ is the maximal $j$ such that $i+(j+1) s-1 \leq m+1$, and

$$
\left[w\left(i+\left(j_{0}+1\right) j\right) \ldots w(m+1)\right]=\left[q(i) \ldots q\left(m+1-\left(i+\left(j_{0}+1\right) s\right)\right)\right],
$$

if $i+\left(j_{0}+1\right) s<m+1$. Again, $w$ is uniquely determined by $q$. Hence, in both cases, $\left|L_{q} \cap C_{i s}^{C}\right| \leq 1$ and, therefore,

$$
\left|L \cap C_{i s}^{C}\right| \leq|Q| \leq\left|\mathcal{W}^{g}(i+s-1)\right|,
$$

which proves the claim.

The above lemma yields the following crucial estimate.

LEMma 5.5. For $m \in \mathbb{N}$,

$$
\left|\mathcal{W}^{g}(m+1)\right| \geq(k-\lfloor\varphi(0)\rfloor)\left|\mathcal{W}^{g}(m)\right|-\sum_{j=1}^{m}(\lfloor\varphi(j)\rfloor-\lfloor\varphi(j-1)\rfloor)\left|\mathcal{W}^{g}(m-j)\right| .
$$

Proof. For $0 \leq j \leq m$, let

$$
H_{j}=\left\{C_{i s} \in \mathcal{C}_{m+1}: i+s-1=m-j\right\},
$$

possibly empty. If $C_{i s} \in H_{j}$, then $i+s+\ell(s)=m+1$ and $i+s-1=m-j$; hence, $\ell(s)=j$. Therefore, $\left|H_{j}\right| \leq|\{s: \ell(s)=j\}|$. We have $\ell(s) \leq j$ if and only if $s \leq \varphi(j)$, and thus

$$
|\{s: \ell(s) \leq j\}|=|\{s: s \leq \varphi(j)\}|=\lfloor\varphi(j)\rfloor .
$$

For $j \geq 1$, this implies that

$$
\begin{aligned}
\left|H_{j}\right| & \leq|\{s: \ell(s)=j\}|=|\{s: \ell(s) \leq j\} \backslash\{s: \ell(s) \leq j-1\}| \\
& =\lfloor\varphi(j)\rfloor-\lfloor\varphi(j-1)\rfloor .
\end{aligned}
$$

Moreover,

$$
|\{s: \ell(s)=0\}|=\left|\left\{s \in \mathbb{N}_{0}: \varphi(0) \geq s\right\}\right|=\lfloor\varphi(0)\rfloor .
$$

Lemma 5.3 concludes the proof.

Finally, we show the existence of a $\varphi$-aperiodic word in $\Sigma^{+}$.

LEMMA 5.6. If condition (3.2) is satisfied, then $\left|\mathcal{W}^{g}(m)\right| \geq c^{m}$. In particular, there exists a $\varphi$-aperiodic word in $\Sigma^{+}$. 
Proof. For $m+1<m_{0}$, we have that $\left|\mathcal{W}^{g}(m+1)\right|=k^{m+1} \geq c^{m+1}$. For $m+1 \geq m_{0}$, assume that $\left|\mathcal{W}^{g}(n)\right| \geq c \cdot\left|\mathcal{W}^{g}(n-1)\right|$ for all $n \leq m$. Then, by the previous lemma,

$$
\begin{aligned}
\left|\mathcal{W}^{g}(m+1)\right| & \geq(k-\lfloor\varphi(0)\rfloor)\left|\mathcal{W}^{g}(m)\right|-\sum_{j=1}^{m}(\lfloor\varphi(j)\rfloor-\lfloor\varphi(j-1)\rfloor)\left|\mathcal{W}^{g}(m-j)\right| \\
& \geq(k-\lfloor\varphi(0)\rfloor)\left|\mathcal{W}^{g}(m)\right|-\sum_{j=1}^{m} \frac{\lfloor\varphi(j)\rfloor-\lfloor\varphi(j-1)\rfloor}{c^{j}}\left|\mathcal{W}^{g}(m)\right| \\
& \geq\left(k-\lfloor\varphi(0)\rfloor-\sum_{j=1}^{\infty} \frac{\lfloor\varphi(j)\rfloor-\lfloor\varphi(j-1)\rfloor}{c^{j}}\right)\left|\mathcal{W}^{g}(m)\right| \geq c \cdot\left|\mathcal{W}^{g}(m)\right|,
\end{aligned}
$$

where we used condition (3.2) in the last inequality. Now Lemma 5.2 implies the existence of a $\varphi$-aperiodic word in $\Sigma^{+}$.

Given a $\varphi$-aperiodic word $w \in \Sigma^{+}$and a letter $a \in \mathcal{A}$, extend $w$ to a word . . aaaw $=$ : $\bar{w} \in \Sigma$ (in the obvious way). Consider the sequence $\left\{T^{n} \bar{w}\right\}_{n \in \mathbb{N}}$ in the compact space $\Sigma$, and let $w_{0}$ be an accumulation point. Note that from the definition of the metric $\bar{d}$, a sequence $w^{n}$ in $\Sigma$ converges to a word $w_{0} \in \Sigma$ if and only if, for every $l \in \mathbb{N}_{0}$, there exists $N \in \mathbb{N}$ such that $\left[w^{n}(-l) \ldots w^{n}(l)\right]=\left[w_{0}(-l) \ldots w_{0}(l)\right]$ for every $n \geq N$. It therefore follows that $\varphi$-aperiodicity is a closed condition (as shown similarly in Lemma 2.4). Since every $T^{n} \bar{w}$ is $\varphi$-aperiodic starting at time $-(n-1), w_{0}$ is a $\varphi$-aperiodic word in $\Sigma$. This proves Theorem 3.3.

5.2. Proof of Theorem 4.6. Recall that $M$ is a closed hyperbolic manifold of dimension $n \geq 2$, and we have $\ln (2)<r_{0}<\bar{\varepsilon}_{0}<i_{M}$. Moreover, $\bar{\varphi}: \mathbb{N}_{0} \rightarrow[0, \infty)$ is a non-decreasing unbounded function for which conditions (4.3) and (4.4) are satisfied with respect to the given minimal shift $\bar{s}_{0} \in \mathbb{N}_{0}$.

A reference for the following is given by $[4,19]$. Let $\mathbb{H}^{n}$ be the $n$-dimensional hyperbolic upper half-space model, where $d$ denotes the hyperbolic distance function on $\mathbb{H}^{n}$. Let $\Gamma$ be the discrete, torsion-free subgroup of the isometry group of $\mathbb{H}^{n}$ identified with the fundamental group $\pi_{1}(M)$ of $M$ acting cocompactly on $\mathbb{H}^{n}$ such that the manifold $\Gamma \backslash \mathbb{H}^{n}$ with an induced smooth and metric structure is isometric to $M$. Let $\pi: \mathbb{H}^{n} \rightarrow$ $\Gamma \backslash \mathbb{H}^{n} \cong M$ be the projection map. Assume all geodesic segments, rays or lines to be parametrized by arc length and identify their images with their point sets in $\mathbb{H}^{n}$. Let $\partial_{\infty} \mathbb{H}^{n}$ be the set of equivalence classes of asymptotic rays in $\mathbb{H}^{n}$, which we identify with the set $\mathbb{R}^{n-1} \cup\{\infty\}$, where $\overline{\mathbb{H}}^{n}-\{\infty\}=\mathbb{H}^{n} \cup \mathbb{R}^{n-1}$ is equipped with the induced Euclidean topology. If $\gamma$ is a ray in $\mathbb{H}^{n}$, we will simply write $\gamma(\infty)$ for the corresponding point in $\partial_{\infty} \mathbb{H}^{n}$. For any two points $p$ and $q$ in $\overline{\mathbb{H}}^{n}$, denote by $[p, q]$ the geodesic segment, ray or line in $\mathbb{H}^{n}$ —depending on whether $p, q \in \mathbb{H}^{n}, p \in \mathbb{H}^{n}$ and $q \in \partial_{\infty} \mathbb{H}^{n}$, or $p, q \in \partial_{\infty} \mathbb{H}^{n}$ respectively-connecting $p$ and $q$.

For $t \in \mathbb{R}$, let $H_{t}:=\mathbb{R}^{n-1} \times\left\{e^{-t}\right\} \subset \mathbb{H}^{n}$. This equals the horosphere based at $\infty$ through the point $\gamma(t)$ of the unit speed geodesic $\gamma(t)=\left(0, e^{-t}\right)$. Let $h_{t}$ be the induced length metric on $H_{t}$ with respect to $d$. The geometry of horospheres in the hyperbolic space is well known; see, for instance, [8] for the following facts. $\left(H_{t}, h_{t}\right)$ is a complete and flat metric space, isometric to the $(n-1)$-dimensional Euclidean space. If $\gamma_{i}: \mathbb{R} \rightarrow \mathbb{H}^{n}$ 


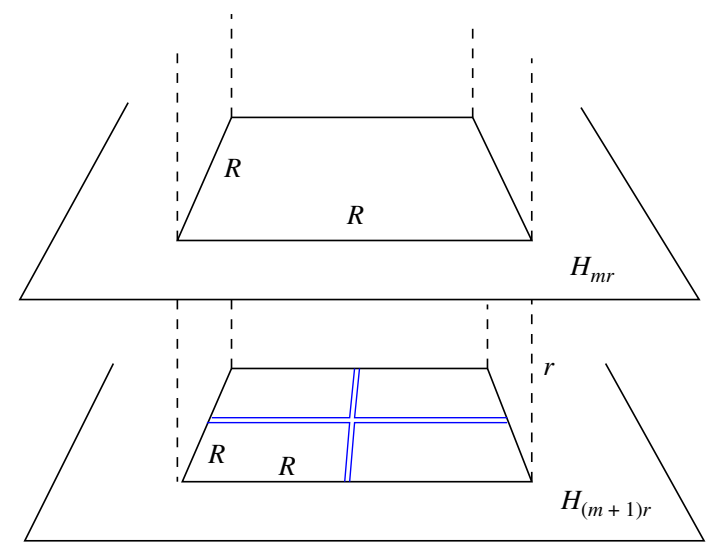

FIGURE 1. Shadowing cubes in horospheres $(n=3)$.

with $\gamma_{i}(0) \in H_{0}, i=1,2$, are two geodesic lines in $\mathbb{H}^{n}$ with $\gamma_{1}(-\infty)=\gamma_{2}(-\infty)=\infty$ and $\gamma_{1}(0), \gamma_{2}(0)$ in the same horosphere, let $\mu(t):=h_{t}\left(\gamma_{1}(t), \gamma_{2}(t)\right)$. Then, for $t \geq 0$,

$$
\mu(t)=e^{t} \mu(0)
$$

Moreover, for two points $p, q$ in the same horosphere $H_{t}$, we have

$$
h_{t}(p, q)=2 \sinh (d(p, q) / 2) .
$$

Now let $\tau>0$ be such that the discretization constant satisfies $r_{0}=\ln 2+\tau$. Let $R>0$ be a fixed length, say $R=1$. Define $Q$ to be an isometric copy of a closed $(n-1)$ dimensional cube $[-R / 2, R / 2]^{n-1}$ of edge lengths $R$ in the Euclidean space $\mathbb{R}^{n-1}$ and contained in the horosphere $H_{0}$. Starting with the cube $Q$ as a reference, we inductively shed shadows in the horospheres $H_{m r_{0}}, m \in \mathbb{N}$, as follows.

Definition 5.7. Given two disjoint sets $S$ and $S^{\prime}$ in $\overline{\mathbb{H}}^{n}$, the set $\mathcal{S}\left(S ; S^{\prime}\right):=\left\{q \in S^{\prime}: S \cap\right.$ $[\infty, q] \neq \emptyset$ is called the shadow of $S$ in $S^{\prime}$ (with respect to $\infty$ ).

By (5.5), the shadow $\mathcal{S}\left(Q ; H_{r_{0}}\right)$ of $Q$ is an isometric copy of a closed $(n-1)$ dimensional cube of edge lengths $e^{r_{0}} R=\left(2+e^{\tau}\right) R$, contained in $H_{r_{0}}$. Hence, there exist $2^{n-1}$ disjoint isometric copies $Q_{j}, j \in\left\{1, \ldots, 2^{n-1}\right\}$, of $Q$ in $\mathcal{S}\left(Q ; H_{r_{0}}\right)$; see Figure 1 .

For $m \geq 1$, let the closed disjoint cubes $Q_{i_{1} \ldots i_{m}}$ in $H_{m r_{0}}$ be already defined. Fix a cube $Q_{i_{1} \ldots i_{m}}$; then, as above, the shadow

$$
\mathcal{S}\left(Q_{i_{1} \ldots i_{m}} ; H_{(m+1) r_{0}}\right) \subset H_{(m+1) r_{0}}
$$

contains $2^{n-1}$ disjoint isometric copies $Q_{i_{1} \ldots i_{m} j}$ of $Q, j \in\left\{1, \ldots, 2^{n-1}\right\}$. Hence, for an alphabet $\mathcal{A}=\left\{1, \ldots, 2^{n-1}\right\}$, we associate a finite word $[w(1) \ldots w(m+1)] \in \Sigma^{+}(m+$ 1) with the cube $Q_{i_{1} \ldots i_{m+1}}$ in $H_{(m+1) r_{0}}$, where $w(n)=i_{n}$, for all $n \in\{1, \ldots, m+1\}$. In particular, we obtain a bijection of finite words $\Sigma^{+}(m)$ of length $m$ with the set of cubes

$$
\mathcal{Q}(m):=\left\{Q_{i_{1} \ldots i_{m}} \subset H_{m r_{0}}: i_{n} \in\left\{1, \ldots, 2^{n-1}\right\} \text { for } 1 \leq n \leq m\right\} .
$$


We denote the closed cubes $Q_{i_{1} \ldots i_{m}}$ obtained in this way by $q(1) \ldots q(m)$, where $q(n) \in$ $\left\{1, \ldots, 2^{n-1}\right\}$ for $n \in\{1, \ldots, m\}$. Every sequence of cubes $\{q(1) q(2) \ldots q(m)\}_{m \in \mathbb{N}}$, successively shadowed from the previous ones, determines a unique point

$$
\eta:=\bigcap_{m \in \mathbb{N}} \mathcal{S}\left(q(1) \ldots q(m) ; \mathbb{R}^{n-1}\right) \in \mathbb{R}^{n-1},
$$

since $\mathcal{S}\left(q(1) \ldots q(m) ; \mathbb{R}^{n-1}\right), m \in \mathbb{N}$, is a sequence of closed nested subsets of $\mathbb{R}^{n-1}$ with diameters converging to 0 . Define $\eta=: q(1) q(2) \ldots$ in $\mathbb{R}^{n-1}$. By construction, the geodesic line $[\infty, \eta]$ runs through every cube $q(1) \ldots q(m), m \in \mathbb{N}$, of the particular sequence. Hence, we obtain a bijection of infinite sequences $q(1) q(2) \ldots$ of cubes and words $w=:[w(1) w(2) \ldots]$ in $\Sigma^{+}$.

Notation. Given a cube $q(1) \ldots q(m)$ in $\mathcal{Q}(m)$, and an integer $n \leq m$, let $\left.q(1) \ldots q(m)\right|_{n} \in$ $\mathcal{Q}(n)$ be the unique cube such that $q(1) \ldots q(m)$ lies in the shadow of $\left.q(1) \ldots q(m)\right|_{n}$. Moreover, for $\xi \in \mathbb{R}^{n}$, we denote the geodesic subsegment $[i, j](\xi)$ by

$$
[i, j](\xi):=\left.[\infty, \xi]\right|_{\left[i r_{0}, j r_{0}\right]}:\left[i r_{0}, j r_{0}\right] \rightarrow \mathbb{H}^{n},
$$

where we assume that $[\infty, \xi](0) \in H_{0}$ and that $i, j \in \mathbb{N}_{0}$, with $i \leq j$, which connects the horospheres $H_{i r_{0}}$ to $H_{j r_{0}}$ and is orthogonal to both. If $i=j$, then we write $[i](\xi):=$ $[i, i](\xi)$, which is the orthogonal projection of $\xi$ on the horosphere $H_{i r_{0}}$.

We again define the admissible set,

$$
A(m):=\left\{(i, s) \in \mathbb{N} \times \mathbb{N}: i+s+\bar{\ell}(s)=m, s>\bar{s}_{0}\right\},
$$

if $m \geq m_{0}:=2+\bar{s}_{0}+\bar{\ell}\left(\bar{s}_{0}+1\right)$, and set $A(m)$ to be empty for $m<m_{0}$.

Definition 5.8. Let $\psi \in \Gamma$ be an isometry and let $i, s \in \mathbb{N}, l \in \mathbb{N}_{0}$. If $\xi \in \mathbb{R}^{n-1}$ such that $d(\psi([i](\xi)),[i+s](\xi))<\bar{\varepsilon}_{0}$, and also $d(\psi([i+l](\xi)),[i+s+l](\xi))<\bar{\varepsilon}_{0}$, we write

$$
\psi([i, i+l](\xi)) \sim_{\bar{\varepsilon}_{0}}[i+s, i+s+l](\xi) .
$$

In particular, by convexity of the distance function, we have, for all $j \in\{0, \ldots, l\}$,

$$
d(\psi([i, i+j](\xi)),[i+s, i+s+j](\xi))<\bar{\varepsilon}_{0} .
$$

We are now able to translate the proof of Theorem 3.3 for the existence of $\varphi$-aperiodic words into the existence of $\varphi$-aperiodic geodesics by counting good cubes.

Definition 5.9. Let $m \in \mathbb{N}$. A cube $q(1) \ldots q(m)$ in $\mathcal{Q}(m)$ is called good if, for every $\xi \in \mathcal{S}\left(q(1) \ldots q(m) ; \mathbb{R}^{n-1}\right)$, every $\psi \in \Gamma$ and every $i \in \mathbb{N}, l \in \mathbb{N}_{0}$, whenever

$$
\psi([i, i+l](\xi)) \sim_{\bar{\varepsilon}_{0}}[i+s, i+s+l](\xi)
$$

for some shift $s>\bar{s}_{0}$ such that $i+s+l \leq m$, then $s>\bar{\varphi}(l)$. Otherwise, $q(1) \ldots q(m)$ is called bad.

If the cube $q(1) \ldots q(m)$ is good, then, since $\bar{\varepsilon}_{0}<i_{M}$, for every $x \in q(1) \ldots q(m)$, the projection of the geodesic segment $\left.[\infty, x]\right|_{\left[r_{0}, m r_{0}\right]}$ into $M$ is $\bar{\varphi}$-aperiodic, up to length $m r_{0}$, with respect to condition (4.2) (see the proof Lemma 5.10(2)). 
Analogously to the proof of Theorem 3.3, for $(i, s) \in A(m)$ and $m \geq m_{0}$, define

$$
\begin{aligned}
C_{i s}:=\{ & q(1) \ldots q(m) \in \mathcal{Q}(m): \text { for all } \xi \in \mathcal{S}\left(q(1) \ldots q(m) ; \mathbb{R}^{n-1}\right) \text { and } \psi \in \Gamma, \\
& \left.\psi([i, i+\bar{\ell}(s)](\xi)) \chi_{\bar{\varepsilon}_{0}}[i+s, m](\xi)\right\},
\end{aligned}
$$

and let $\mathcal{C}_{m}$ be the set of all $C_{i j}$ for $(i, j) \in A(m)$. Note that $\mathcal{C}_{m}$ is empty if $m<m_{0}$.

With respect to these definitions, the relationship between Definitions 4.4 and 5.9, respectively, and the sets $C_{i s}$ is given by the following lemma.

LEMMA 5.10.

(1) For $m<m_{0}$, every cube $q(1) \ldots q(m) \in \mathcal{Q}(m)$ is good. For $m \geq m_{0}$, the cube $q(1) \ldots q(m) \in \mathcal{Q}(m)$ is good if $\left.q(1) \ldots q(m)\right|_{n} \in C_{i s}$, for all $n \leq m$ and $(i, s) \in$ $A(n)$.

(2) Let $q(1) q(2) \ldots$ be an infinite sequence of cubes and let $\eta \in \mathbb{R}^{n-1}$ be the unique corresponding limit point. The discrete geodesic $\overline{\pi \circ\left[r_{0}, \infty\right)(\eta)}$ in $M$ is $\bar{\varphi}$-aperiodic at every time $i \in \mathbb{N}$ if, for all $m \in \mathbb{N}$ and $(i, s) \in A(m)$, the cube $q(1) \ldots q(m)$ in $\mathcal{Q}(m)$ of the sequence $q(1) q(2) \ldots$ belongs to $C_{i s}$.

Proof. For (1), first let $m<m_{0}$. Let $i, s \in \mathbb{N}, l \in \mathbb{N}_{0}$ such that $s>\bar{s}_{0}$ and $i+s+l \leq m<$ $2+\bar{s}_{0}+\bar{\ell}\left(\bar{s}_{0}+1\right)$. In particular, $l<\bar{\ell}\left(\bar{s}_{0}+1\right)$, so that $\varphi(l)<\bar{s}_{0}+1 \leq s$, and every cube $q(1) \ldots q(m)$ follows to be good.

Now let $m \geq m_{0}$. Assume for contradiction that $q(1) \ldots q(m)$ is not good and let $\xi \in \mathcal{S}\left(q(1) \ldots q(m) ; \mathbb{R}^{n-1}\right)$ and $\psi \in \Gamma$ such that for some $i \in \mathbb{N}, l \in \mathbb{N}_{0}$, we have

$$
\psi([i, i+l](\xi)) \sim_{\bar{\varepsilon}_{0}}[i+s, i+s+l](\xi),
$$

where $s>\bar{s}_{0}$ with $i+s+l \leq m$ and $s \leq \bar{\varphi}(l)$. Hence, $\bar{\ell}(s) \leq l$, and for $n:=i+s+\bar{\ell}(s)$, we have, in particular, by (5.7),

$$
\psi([i, i+\bar{\ell}(s)](\xi)) \sim_{\bar{\varepsilon}_{0}}[i+s, n](\xi) .
$$

Hence, we see that $\left.q(1) \ldots q(m)\right|_{n} \notin C_{i s}$, where $(i, s) \in A(n)$ for $n \leq m$; a contradiction.

For (2), assume that $\bar{\gamma}:=\overline{\pi \circ\left[r_{0}, \infty\right)(\eta)}$ is not $\bar{\varphi}$-aperiodic at time $i \in \mathbb{N}$. Then there must be a shift $s \in \mathbb{N}$ with $s>\bar{s}_{0}$, and $l \in \mathbb{N}_{0}$, such that

$$
d(\bar{\gamma}(i+j), \bar{\gamma}(i+s+j))<\bar{\varepsilon}_{0} \quad \text { for all } j \in\{0, \ldots, l\},
$$

where $s \leq \bar{\varphi}(l)$. Since $\bar{\varepsilon}_{0}<i_{M}$ and the distance function is convex, we also have $d\left(\gamma\left((i+t) r_{0}\right), \gamma\left((i+s+t) r_{0}\right)\right)<\bar{\varepsilon}_{0}$, for all $0 \leq t \leq l$, for the corresponding extended geodesic $\gamma: \mathbb{R} \rightarrow M$. By the discreteness of $\Gamma$, there exist finitely many isometries $\psi_{1}, \ldots, \psi_{q} \in \Gamma$ and a subdivision of the interval $\left[i r_{0},(i+l) r_{0}\right]$ into $\left[l_{0} r_{0}, l_{1} r_{0}\right],\left[l_{1} r_{0}, l_{2} r_{0}\right], \ldots,\left[l_{q-1} r_{0}, l_{q} r_{0}\right]$, where $l_{0}=i$ and $l_{q}=i+l$ and $l_{j} \in \mathbb{R}$, such that (with analogous notation as above)

$$
\psi_{j+1}\left(\left[l_{j}, l_{j+1}\right](\eta)\right) \sim_{\bar{\varepsilon}_{0}}\left[s+l_{j}, s+l_{j+1}\right](\eta), \quad j=0, \ldots, q-1 .
$$

We thus have $d\left(\psi_{j+1}\left(\left[l_{j+1}\right](\eta)\right),\left[s+l_{j+1}\right](\eta)\right)<\bar{\varepsilon}_{0}$ and $d\left(\psi_{j+2}\left(\left[l_{j+1}\right](\eta)\right),[s+\right.$ $\left.\left.l_{j+1}\right](\eta)\right)<\bar{\varepsilon}_{0}$. Since $\bar{\varepsilon}_{0}<i_{M}$ and every orbit of $\Gamma$ is $2 i_{M}$-separated (that is, for $\psi$, $\bar{\psi} \in \Gamma$, we have $d(\psi x, \bar{\psi} x) \geq 2 i_{M}$ for any $\left.x \in \mathbb{H}^{n}\right)$, it follows from the triangle inequality 
that $\psi_{j+1}\left(\left[l_{j+1}\right](\eta)\right)=\psi_{j+2}\left(\left[l_{j+1}\right](\eta)\right)$; hence, $\psi_{j+1}=\psi_{j+2}$ for all $j=0, \ldots, q-2$, since $\Gamma$ acts freely. Therefore, we have an isometry $\psi \in \Gamma$ such that

$$
\psi([i, i+l](\eta)) \sim_{\bar{\varepsilon}_{0}}[i+s, i+s+l](\eta),
$$

where $s \leq \bar{\varphi}(l)$. The proof is now finished analogously to the case of (1).

In view of Lemma 5.10, let, for $m \geq m_{0}$,

$$
\mathcal{Q}^{g}(m)=\left\{q(1) \ldots q(m) \in \mathcal{Q}(m):\left.q(1) \ldots q(m)\right|_{n} \in C_{i s} \text { for all }(i, s) \in A(n), n \leq m\right\},
$$

and $Q^{g}(m)=\mathcal{Q}(m)$ for $m<m_{0}$, which is a subset of all good cubes at step $m$.

Lemma 5.11. Assume that condition (4.3) is satisfied. Then, for $m \in \mathbb{N}$,

$$
\left|\mathcal{Q}^{g}(m+1)\right| \geq k\left|\mathcal{Q}^{g}(m)\right|-\bar{c} \cdot \sum_{C_{i s} \in \mathcal{C}_{m+1}}\left|\mathcal{Q}^{g}(i+s-1)\right|,
$$

where $\bar{c}$ is a constant depending only on $n, i_{M}$ and $\bar{s}_{0}$, and is strictly decreasing in $\bar{s}_{0}$.

Proof. If $m+1<m_{0}$, then $\mathcal{C}_{m+1}$ is empty and the claim follows. Hence, assume $m+1 \geq$ $m_{0}$. Let

$$
L=\left\{q(1) \ldots q(m+1) \in \mathcal{Q}(m+1):\left.q(1) \ldots q(m+1)\right|_{m} \in \mathcal{Q}^{g}(m)\right\},
$$

and note that $|L|=k\left|Q^{g}(m)\right|$. Then

$$
\mathcal{Q}^{g}(m+1)=L \cap\left(\bigcap_{C_{i s} \in \mathcal{C}_{m+1}} C_{i s}\right)=L \backslash\left(\bigcup_{C_{i s} \in \mathcal{C}_{m+1}}\left(L \cap C_{i s}^{C}\right)\right),
$$

where $C_{i s}^{C}$ is the complement of $C_{i s}$. Fix some $C=C_{i s} \in \mathcal{C}_{m+1}$. Define

$$
Q=\left\{\left.q(1) \ldots q(m+1)\right|_{i+s-1} \in \mathcal{Q}(i+s-1): q(1) \ldots q(m+1) \in L\right\} .
$$

One checks that $|Q| \leq\left|\mathcal{Q}^{g}(i+s-1)\right|$. Let $L=\bigcup_{q \in Q} L_{q}$, where

$$
L_{q}=\left\{q(1) \ldots q(m+1) \in L:\left.q(1) \ldots q(m)\right|_{i+s-1}=q\right\} .
$$

It remains to show that each $L_{q} \cap C^{C}$ contains at most $\bar{c}$ cubes; in this case,

$$
\left|L \cap C^{C}\right| \leq \bar{c} \cdot|Q| \leq \bar{c} \cdot\left|\mathcal{Q}^{g}(i+s-1)\right| .
$$

The following claim concludes the proof.

Claim 5.12. $\left|L_{q} \cap C^{C}\right| \leq \bar{c} \cdot\left|\mathcal{Q}^{g}(i+s-1)\right|$.

For the proof of the claim, note that if (4.4) is satisfied, then for all $l \geq \bar{s}_{0}$,

$$
\lfloor\bar{\varphi}(l)\rfloor>l,
$$

which implies that for all $s>\bar{s}_{0}$,

$$
\bar{\ell}(s)<s .
$$

To see this, assume $\bar{\ell}(s) \geq s$ for some $s>\bar{s}_{0}$. Then, by definition of $\bar{\ell}, \bar{\varphi}(j)<s$ for all $s>j \in \mathbb{N}_{0}$. In particular, for $\bar{s}_{0}<s$, we have $\bar{\varphi}\left(\bar{s}_{0}\right) \geq\left\lfloor\bar{\varphi}\left(\bar{s}_{0}\right)\right\rfloor$; a contradiction to $\left\lfloor\bar{\varphi}\left(\bar{s}_{0}\right)\right\rfloor>\bar{s}_{0}$. 


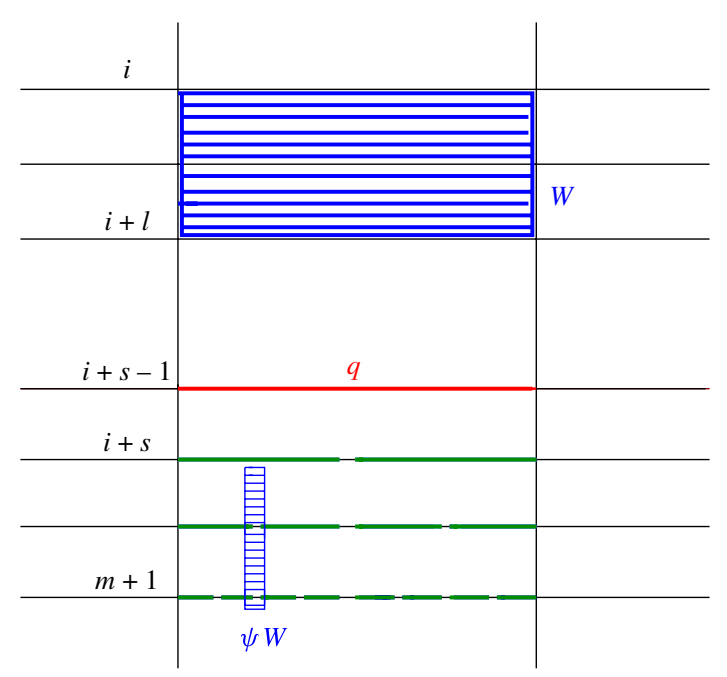

FIGURE 2. Cubes which become bad by the isometry $\psi(n=2)$.

Proof of Claim 5.12. $L_{q}$ consists of cubes of the form $q \cdot q(i+s) \ldots q(m+1) \in \mathcal{Q}(m+$ 1). Hence, consider the point set $W$ of all geodesic segments $[i, i+\bar{\ell}(s)](\xi)$, where $\xi \in \mathcal{S}\left(q, \mathbb{R}^{n-1}\right)$; see Figure 2. Since $s>\bar{s}_{0}$, we have $\bar{\ell}(s)<s$, by (5.10), and therefore $s-1-\bar{\ell}(s) \geq 0$. Moreover, by definition, the cube $q$ in $H_{(i+s-1) r_{0}}$ has $h$-edge lengths $R$. Thus, from (5.5), the subset $H_{i+\bar{\ell}(s)} \cap W$ is isometric to a Euclidean cube with $h$-edge length

$$
e^{-(i+s-1) r_{0}+(i+\bar{\ell}(s)) r_{0}} R=e^{-(s-1-\bar{\ell}(s)) r_{0}} R \leq R .
$$

Since a Euclidean cube in $\mathbb{R}^{n-1}$ of edge length $L$ has diameter at most $\sqrt{n-1} L$, we obtain from (5.6) that the $d$-diameter of $H_{i+\bar{\ell}(s)} \cap W$ is bounded above by

$$
2 \operatorname{arcsinh}\left(e^{-(s-1-\bar{\ell}(s)) r_{0}} \sqrt{n-1} R / 2\right) .
$$

In the same way, the $h$-edge length of $H_{i r_{0}} \cap W$ is given by

$$
e^{-(s-1) r_{0}} R \text {. }
$$

Now, by definition, for every $q \cdot q(i+s) \ldots q(m+1) \in L_{q} \cap C^{C}$, there exists $\psi \in$ $\Gamma$ such that $\psi([i, i+\bar{\ell}(s)](\xi)) \sim_{\bar{\varepsilon}_{0}}[i+s, m+1](\xi)$ for some $\xi \in \mathcal{S}\left(q, \mathbb{R}^{n-1}\right)$. In particular, $x:=[m+1](\xi)$ must belong to the $\bar{\varepsilon}_{0}$-neighborhood of $\psi\left(W \cap H_{i+s+\bar{\ell}(s)}\right)$. Thus, we want to estimate the maximal number of cubes in $\mathcal{Q}(m+1)$ which intersect with the $\bar{\varepsilon}_{0}$-neighborhood of $\psi\left(W \cap H_{i+s+\bar{\ell}(s)}\right)$. Therefore, also let $y \in H_{(m+1) r_{0}}$ belong to the $\bar{\varepsilon}_{0}$-neighborhood of $\psi\left(W \cap H_{i+s+\bar{\ell}(s)}\right)$. By the triangle inequality and by (5.11), we have

$$
d(x, y) \leq 2 \bar{\varepsilon}_{0}+2 \operatorname{arcsinh}\left(e^{-(s-1-\bar{\ell}(s)) r_{0}} \sqrt{n-1} R / 2\right) .
$$

Therefore, again from (5.6), the $h$-diameter of the intersection of the $\bar{\varepsilon}_{0}$-neighborhood of $\psi\left(W \cap H_{i+s+\bar{\ell}(s)}\right)$ with $H_{(m+1) r_{0}}$ is bounded above by

$$
\bar{r}_{1}(s):=2 \sinh \left(\bar{\varepsilon}_{0}+\operatorname{arcsinh}\left(e^{-(s-1-\bar{\ell}(s)) r_{0}} \sqrt{n-1} R / 2\right)\right) .
$$


On the other hand, the cubes $q \cdot q(i+s) \ldots q(m+1) \in \mathcal{Q}(m+1)$ are disjoint and have Euclidean volume $R^{n-1}$. Therefore, we set

$$
\bar{c}_{1}(s):=\left\lceil\frac{\left(\bar{r}_{1}(s)+\sqrt{n-1} R\right)^{n-1}}{R^{n-1}}\right\rceil .
$$

Hence, the $\bar{\varepsilon}_{0}$-neighborhood of $\psi\left(W \cap H_{i+s+\bar{\ell}(s)}\right)$ can intersect at most $\bar{c}_{1}(s)$ cubes in $\mathcal{Q}(m+1)$. Since $q(1) \ldots q(m)$ is good for every $q(1) \ldots q(m+1) \in L_{q}$, we conclude that, with respect to $\psi$, at most $\bar{c}_{1}(s)$ cubes can become bad in $L_{q} \cap C^{C}$.

Now let $\bar{y}$ be the center of $W \cap H_{i r_{0}}$, which is isometric to a cube in the Euclidean space of edge length $e^{-(s-1) r_{0}} R$, by (5.12), and contained in the cube $\left.q\right|_{i}$. From (5.6), $W \cap H_{i r_{0}}$ must be contained in the hyperbolic ball $B_{d}\left(\bar{y}, \bar{r}_{2}(s)\right)$, where

$$
\bar{r}_{2}(s)=2 \operatorname{arcsinh}\left(e^{-(s-1) r_{0}} \sqrt{n-1} R / 4\right) .
$$

Note that if there is some point $p \in W \cap H_{i r_{0}}$ and some $\psi \in \Gamma$ such that $d(\psi p, \bar{q})<$ $\bar{\varepsilon}_{0}$, where $\bar{q}:=\mathcal{S}\left(q, H_{(i+s) r_{0}}\right)$, then $d(\psi \bar{y}, \bar{q})<\bar{\varepsilon}_{0}+\bar{r}_{2}(s)$. In particular, for every cube $q \cdot q(i+s) \ldots q(m+1) \in L_{q} \cap C^{C}$, there exists such an isometry $\psi$. However, since the orbit $\Gamma \bar{y}$ is $2 i_{M}$-separated, the open metric balls $B\left(\psi \bar{y}, i_{M}\right), \psi \in \Gamma$, are disjoint, and there can only be finitely many, say $\bar{c}_{2}(j)$, intersecting the $\max \left\{\bar{\varepsilon}_{0}+\right.$ $\left.\bar{r}_{2}(s)-i_{M}, 0\right\}$-neighborhood of $\bar{q}$. In fact, from (5.5) and (5.6), the $h$-diameter of $\bar{q}$ is bounded above by $e^{r_{0}} \sqrt{n-1} R$, and $\bar{q}$ must be contained in a hyperbolic ball of radius $2 \operatorname{arcsinh}\left(e^{r_{0}} \sqrt{n-1} R / 4\right)$. Therefore, $\bar{c}_{2}(s)$ is bounded above by

$$
\left\lceil\frac{\operatorname{vol}\left(B\left(2 \operatorname{arcsinh}\left(e^{r_{0}} \sqrt{n-1} R / 4\right)+2 \operatorname{arcsinh}\left(e^{-(s-1) r_{0}} \sqrt{n-1} R / 4\right)+\bar{\varepsilon}_{0}\right)\right)}{\operatorname{vol}\left(B\left(i_{M} / 2\right)\right)}\right\rceil .
$$

Since both $\bar{c}_{1}(s)$ and $\bar{c}_{2}(s)$ are non-increasing in $s$, we conclude the claim by setting $\bar{c}:=\bar{c}_{1}\left(\bar{s}_{0}+1\right) \bar{c}_{2}\left(\bar{s}_{0}+1\right)$.

Analogously to the proof of Lemma 5.5, the previous lemma yields the following.

Lemma 5.13. Assume that condition (5.10) is satisfied. Then, for $m \in \mathbb{N}$,

$$
\begin{aligned}
\left|\mathcal{Q}^{g}(m+1)\right| \geq & \left(k-\mathbf{1}_{\left\{\bar{\ell}\left(\bar{s}_{0}+1\right)=0\right\}} \bar{c}\lfloor\bar{\varphi}(0)\rfloor\right)\left|\mathcal{Q}^{g}(m)\right| \\
& -\bar{c} \cdot \sum_{j=\max \left\{\bar{\ell}_{\left.\left(s_{0}+1\right), 1\right\}}\right.}^{m}(\lfloor\bar{\varphi}(j)\rfloor-\lfloor\bar{\varphi}(j-1)\rfloor)\left|\mathcal{Q}^{g}(m-j)\right| .
\end{aligned}
$$

Proof. Recall the definition of the set $H_{j}=\left\{C_{i s} \in \mathcal{C}_{m+1}: i+s-1=m-j\right\}$ in (5.3). Since $\bar{\ell}$ is non-decreasing, we have $j=m+1-(i+s)=\bar{\ell}(s) \geq \bar{\ell}\left(\bar{s}_{0}+1\right)$ if $s>\bar{s}_{0}$.

Finally, if, moreover, condition (4.4) is satisfied, then the same inductive proof as in Lemma 5.6 shows that the number of good cubes in $Q^{g}(m+1)$ increases in $m+1$ by the factor $c>1$; see (5.4). Lemma 5.10(2) then shows the existence of a $\bar{\varphi}$-aperiodic geodesic $\bar{\gamma}: \mathbb{N} \rightarrow M$. Thus, we have shown the following lemma.

LEMmA 5.14. Assume that conditions (4.3) and (4.4) are satisfied. Then, for $m \in$ $\mathbb{N},\left|\mathcal{Q}^{g}(m)\right| \geq c^{m}$. In particular, there exists a $\bar{\varphi}$-aperiodic geodesic $\bar{\gamma}: \mathbb{N} \rightarrow M$ with parameters $\left(\bar{s}_{0}, \bar{\varepsilon}_{0}, r_{0}\right)$. 
Now let $\bar{\gamma}: \mathbb{N} \rightarrow M$ be a $\bar{\varphi}$-aperiodic geodesic (with parameters $\left(\bar{s}_{0}, \bar{\varepsilon}_{0}, r_{0}\right)$ ), and let $\gamma$ : $\mathbb{R} \rightarrow M$ be the corresponding extended geodesic. Consider the sequence $v^{n}:=\phi^{n} \gamma^{\prime}\left(r_{0}\right)$, $n \in \mathbb{N}$, in the compact space $S M$, and let $\gamma_{0}$ be an accumulation point. The space of unit speed geodesics (identified with $S M$ ) is endowed with the topology of uniform convergence on bounded sets. Therefore, note that a sequence $v^{n}$ converges to $v$ in $S M$ if and only if, for every $l \geq 0$ and every $\tau>0$, there exists $N \in \mathbb{N}$ such that for every $n \geq N, d\left(\gamma_{v^{n}}(t), \gamma_{v}(t)\right)<\tau$ for every $t \in[-l, l]$. Therefore, $\bar{\varphi}$-aperiodicity can be shown to be a closed condition (similarly as in Lemma 2.4). Since $\bar{\gamma}_{v^{n}}$ is $\bar{\varphi}$-aperiodic beginning at $t_{n} \geq-(n-1)$ (with parameters $\left(\bar{s}_{0}, \bar{\varepsilon}_{0}, r_{0}\right)$ ), it follows that $\bar{\gamma}_{0}: \mathbb{Z} \rightarrow M$ is $\bar{\varphi}$-aperiodic. This completes the proof of Theorem 4.6.

5.3. Proof of Theorem 4.3. For $\delta \in(0,1)$, choose $\bar{\delta} \in[\delta, 1)$ such that for $r_{0}=\ln (3-\bar{\delta})$, we have $\ln (3-\bar{\delta})+\varepsilon_{0}<i_{M}$. Note that $\tilde{\delta}=\bar{\delta} \ln (2) / \ln (3-\bar{\delta}) \rightarrow 1$ as $\bar{\delta} \rightarrow 1$, and assume therefore that $\tilde{\delta}>\delta$. For $l \geq 0$, let $\bar{\psi}(l)=2^{\bar{\delta}(n-1) l}$, so that its right inverse $\lceil(1 / \bar{\delta}(n-$ 1) $\ln (2)) \ln (s)\rceil$ is an unbounded function. Then, for $c=\frac{1}{2}\left(2^{n-1}+2^{\bar{\delta}(n-1)}\right)$, we have that for sufficiently large $\bar{s}_{0}=\bar{s}_{0}\left(\bar{\delta}, n, i_{M}, \varepsilon_{0}\right) \in \mathbb{N}_{0}$, the conditions (4.3) and (4.4) are satisfied. Thus, from Theorem 4.6, there exists a discrete geodesic $\bar{\gamma}: \mathbb{Z} \rightarrow M$ which is $\bar{\psi}$-aperiodic with respect to $\left(\bar{s}_{0}, r_{0}+\varepsilon_{0}, r_{0}\right)$. From Lemma 4.5 , we obtain that $\gamma: \mathbb{R} \rightarrow M$ is continuously $\psi$-aperiodic with parameters $s_{0}=\left(\bar{s}_{0}+1\right) r_{0}$ and $\varepsilon_{0}$, where, for $l \geq r_{0}$,

$$
\begin{aligned}
\psi(l) & =\ln (3-\bar{\delta}) \cdot \bar{\psi}\left(\frac{l}{\ln (3-\bar{\delta})}-1\right)-\ln (3-\bar{\delta}) \\
& =\frac{\ln (3-\bar{\delta})}{2^{\bar{\delta}(n-1)}} e^{(\bar{\delta} \ln (2) / \ln (3-\bar{\delta}))(n-1) l}-\ln (3-\bar{\delta}) \\
& =\left(\frac{\ln (3-\bar{\delta})}{2^{\bar{\delta}(n-1)}}-\frac{\ln (3-\bar{\delta})}{e^{\tilde{\delta}(n-1) l}}\right) e^{\tilde{\delta}(n-1) l} \\
& =: c(\tilde{\delta}, l) \cdot e^{\tilde{\delta}(n-1) l}=c(\tilde{\delta}, l) \varphi_{\tilde{\delta}}(l) .
\end{aligned}
$$

Note that $c(\tilde{\delta}, l)$ is increasing in $l$ and we restrict $\psi$ to the interval $\left[l_{1}, \infty\right)$ for some $l_{1}>\ln (3-\bar{\delta})$ such that $c\left(\tilde{\delta}, l_{1}\right)>0$.

We now translate the minimal shift $s_{0}$ into a sufficiently large minimal length $l_{0} \geq l_{1}$. Assume that for some $t_{0}$ and $s>\varepsilon_{0}$ we have $d\left(\gamma\left(t_{0}+t\right), \gamma\left(t_{0}+s+t\right)\right)<\varepsilon_{0}$ for all $0 \leq t \leq l$, where $l \geq l_{0}$.

First, assume that $s \leq s_{0}$. The closing lemma implies the existence of a closed geodesic nearby; in fact, the following lemma can be shown using standard arguments in hyperbolic geometry.

LEMMA 5.15. In this setting, there exists a closed geodesic $\alpha$ of period $p \leq s+\varepsilon_{0}$ and a constant $s^{\prime}=s^{\prime}\left(s_{0}, \varepsilon_{0}, i_{M}\right)$ such that (up to parametrization of $\alpha$ ),

$$
d\left(\alpha(t), \gamma\left(t_{0}+s^{\prime}+t\right)\right)<\varepsilon_{0} / 2 \text { for all } 0 \leq t \leq s+l-2 s^{\prime} .
$$

Let $N^{\prime}=\left\lceil s_{0} / p\right\rceil \in \mathbb{N}$ be the smallest integer such that $N^{\prime} p>s_{0}$. Then

$$
\begin{aligned}
& d\left(\gamma\left(t_{0}+s^{\prime}+t\right), \gamma\left(t_{0}+s^{\prime}+N^{\prime} p+t\right)\right) \\
& \quad \leq d\left(\gamma\left(t_{0}+s^{\prime}+t\right), \alpha(t)\right)+d\left(\gamma\left(t_{0}+s^{\prime}+N^{\prime} p+t\right), \alpha(t)\right)<\varepsilon_{0}
\end{aligned}
$$


for all $0 \leq t \leq s+l-2 s^{\prime}-N^{\prime} p$. Thus,

$$
N^{\prime} p>c\left(\tilde{\delta}, l_{1}\right) \varphi_{\tilde{\delta}}\left(l-2 s^{\prime}-N^{\prime} p\right)=\frac{c\left(\tilde{\delta}, l_{1}\right)}{e^{\tilde{\delta}(n-1)\left(2 s^{\prime}+N^{\prime} p\right)}} \varphi_{\tilde{\delta}}(l) .
$$

Since $N^{\prime} p \leq c s$ and hence $2 s^{\prime}+N^{\prime} p \leq 2 s^{\prime}+c s_{0}$, for some $c=c\left(s_{0}, \varepsilon_{0}, i_{M}\right)$, we can find a positive constant $c_{0}=c_{0}\left(\tilde{\delta}, i_{M}, n, \varepsilon_{0}\right)$ such that $s>c_{0} \varphi_{\tilde{\delta}}(l)$.

In the case when $s>s_{0}$, we have

$$
s>c\left(\tilde{\delta}, l_{1}\right) \varphi_{\tilde{\delta}}(l) \geq c_{0} \varphi_{\tilde{\delta}}(l) .
$$

Finally, since $\delta<\tilde{\delta}$, we restrict if necessary to $\tilde{l}_{0} \geq l_{0}$ such that $c_{0} \varphi_{\tilde{\delta}}(l) \geq \varphi_{\delta}(l)$ for all $l \geq \tilde{l}_{0}$. This finishes the proof of the theorem.

Acknowledgements. We want to thank Shahar Mozes for helpful discussions. The second author would like to thank Jean-Claude Picaud for many fruitful discussions and comments, and the University of Tours for its hospitality during his stay in January 2012. The authors acknowledge support by the Swiss National Science Foundation (grant no. 135091).

\section{REFERENCES}

[1] V. I. Bernik and M. M. Dodson. Metric Diophantine Approximation on Manifolds. Vol. 137. Cambridge University Press, Cambridge, 1999.

[2] M. D. Boshernitzan. Quantitative recurrence results. Invent. Math. 112 (1993), 617-631.

[3] M. R. Bridson and A. Haeflinger. Metric Spaces of Non-positive Curvature. Springer, Berlin, 1999.

[4] P. Eberlein. Geometry of Nonpositively Curved Manifolds. University of Chicago Press, Chicago, 1994.

[5] M. Einsiedler and T. Ward. Ergodic Theory: with a view towards Number Theory. Springer, London, 2011.

[6] H. Furstenberg. Recurrence in Ergodic Theory and Combinatorial Number Theory. Vol. 2. Princeton University Press, Princeton, NJ, 1981.

[7] A. Haas. Geodesic cusp excursions and metric diophantine approximation. Math. Res. Lett. 16 (2009), 67-85.

[8] E. Heintze and H. C. Im Hof. Geometry of horospheres. J. Differential Geom. 12(4) (1977), 481-491.

[9] S. Hersonsky and F. Paulin. Diophantine approximation for negatively curved manifolds. Math. Z. 241 (2002), 181-226.

[10] S. Hersonsky and F. Paulin. On the almost sure spiraling of geodesics in negatively curved manifolds. J. Differential Geom. (2) 85 (2010), 271-314.

[11] M. Morse and G. Hedlund. Unending chess, symbolic dynamics and a problem in semigroups. Duke Math. J. 11 (1944), 1-7.

[12] D. Ornstein and B. Weiss. Entropy and recurrence rates for stationary random fields. IEEE Trans. Inform. Theory 48(6) (2002), 1694-1697.

[13] J. Parkkonen and F. Paulin. Prescribing the behaviour of geodesics in negative curvature. Geom. Topol. 14 (2010), 277-392.

[14] J. Parkkonen and F. Paulin. Spiraling spectra of geodesic lines in negatively curved manifolds. Math. Z. 268 (2011), 101-142.

[15] S. J. Patterson. Diophantine approximation in Fuchsian groups. Philos. Trans. R. Soc. Lond. Ser. A 282 (1976), 527-563.

[16] J. C. Robinson. Dimensions, Embeddings and Attractors. Vol. 186. Cambridge University Press, Cambridge, 2011. 
[17] D. Sullivan. Disjoint spheres, approximation by imaginary quadratic numbers, and the logarithm law for geodesics. Acta Math. 149 (1982), 215-237.

[18] S. J. Velani. Diophantine approximation and Hausdorff-dimension in Fuchsian groups. Math. Proc. Cambridge Philos. Soc. 113 (1993), 343-354.

[19] W. Ballmann, M. Gromov and V. Schroeder. Manifolds of Nonpositive Curvature. Vol. 61. Birkhäuser, Boston, 1985.

[20] P. Walters. Introduction to Ergodic Theory. Vol. 79. Springer, New York, 1981. 